DOI: $10.1016 / j$. knosys.2010.01.005

Elsevier, 2010

\title{
Using Argumentation to Model and Deploy Agent-based $\mathcal{B} 2 \mathcal{B}$ Applications
}

\author{
Jamal Bentahar ${ }^{\mathrm{a}}$, Rafiul Alam ${ }^{\mathrm{a}}$, Zakaria Maamar ${ }^{\mathrm{b}}$, and Nanjangud C. Narendra ${ }^{\mathrm{c}}$ \\ ${ }^{a}$ Concordia University, Montreal, Canada \\ ${ }^{\mathrm{b}}$ Zayed University, Dubai, U.A.E \\ ${ }^{c}$ IBM India Research Lab, Bangalore, India
}

\begin{abstract}
This paper presents an agent-based framework for modeling and deploying $\mathcal{B}$ usiness-to- $\mathcal{B}$ usiness $(\mathcal{B} 2 \mathcal{B})$ applications, where autonomous agents act on behalf of the individual components that form these applications. This framework consists of three levels identified by strategic, application, and resource, with focus in this paper on the first two levels. The strategic level is about the common vision that independent businesses define as part of their decision of partnership. The application level is about the business processes that get virtually combined as result of this common vision. As conflicts are bound to arise among the independent applications/agents, the framework uses a formal model based on computational argumentation theory through a persuasion protocol to detect and resolve these conflicts. In this protocol, agents reason about partial information using partial arguments, partial attack, and partial acceptability. Agents can then jointly find arguments that support a new solution for their conflicts, which is not known by any of them individually. Termination, soundness, and completeness properties of this protocol are provided. Distributed and centralized coordination strategies are also supported in this framework, which is illustrated with an online-purchasing example.
\end{abstract}

Keywords. $\mathcal{B} 2 \mathcal{B}$, argumentation theory, agent communication, conflict, persuasion.

\section{Introduction}

Today's challenges in terms of performance and competitiveness are putting businesses under a constant pressure of meeting changing requirements. This fuels the need for continuous merge and sometimes re-engineering of business processes, resulting in $\mathcal{B}$ usiness-to- $\mathcal{B}$ usiness $(\mathcal{B} 2 \mathcal{B})$ applications development. Briefly, a $\mathcal{B} 2 \mathcal{B}$ application is a set of business processes that make disparate autonomous entities (e.g., departments, businesses) collaborate to achieve a common set of goals. Despite the abundance of initiatives on $\mathcal{B} 2 \mathcal{B}$ applications $[15,19,26,28]$, not much exist in terms of modeling and deploying such applications from the perspective of using intelligent and argumentativeagents. By modeling, we mean identifying all the necessary components that connect assets of independent entities that are engaged in a $\mathcal{B} 2 \mathcal{B}$ scenario. By deployment, we mean identifying all the necessary technologies that allow the connection of these assets happen effectively. Finally, by argumentation, we mean making agents that represent and operate on behalf of businesses comply with a dialectical process to affirm or dis- 
avow the conclusions that these agents wish to reciprocally convey to their peers [4,5]. In a $\mathcal{B} 2 \mathcal{B}$ scenario, argumentation would broadly assist businesses, through representative agents, engage in intense negotiation and persuasion sessions prior to making any joint decisions ${ }^{1}$. The argumentation capability of an agent representing a business, can assist this business in negotiating with its peers during a conflict situation and in collaborating with them to achieve agreements on their strategies. Although argumentation has been extensively investigated in the last decade and many interesting frameworks are proposed [3], limited efforts have been put into using this theory in concrete applications [23]. This paper addresses this challenging issue, which consists of using argumentation theory for multi-agent systems to develop $\mathcal{B} 2 \mathcal{B}$ applications. In terms of validation, theoretical proofs of some properties are provided and a concrete case study from the industry illustrates the proposed framework. This framework is an initiative within the emerging field of developing intelligent software [11,13]. Many approaches in this field are based on new software development techniques such as the Lyee calculus and methodology [14]. Our approach is different; it uses argumentation theory and agent technology.

This framework suggests three levels, resource, application, and strategic ${ }^{2}$ that are connected through rely-on and run-on-top-of relations (Fig. 1). These levels represent the way businesses generally function: the strategic level, associated with a set of $\mathcal{S}$ trategic Argumentative Agents $(\mathcal{S}$-AAs), sets the goals to reach (e.g., $10 \%$ revenue increase), the application level, associated with a set of $\mathcal{A}$ pplication Argumentative Agents ( $\mathcal{A}$-AAs), sets the automatic and manual processes (e.g., new auditing system) that permit fulfilling these objectives, and the resource level, associated with a set of $\mathcal{R}$ esource Argumentative Agents ( $\mathcal{R}$-AAs), sets the means that achieve the performance of these processes. The framework associates components (that reside in one of the three levels) with agents equipped with argumentation capabilities to assist a specific component (i) persuade peers of collaborating, (ii) interact with peers during business process implementation, (iii) agree with peers on the collaboration outcomes, (iv) resolve conflicts that could impede collaboration, and (v) track conflict resolution. Still in Fig. 1, the rely-on relation means mapping the business goals onto concrete system applications, and run-on-top-of relation means performing these system applications' business processes subject to resource availabilities. In addition, both relations make issues at lower levels influence goals at higher levels. For example, lack of resources could result in reviewing expansion goals.

In Fig. 1, horizontal relations permit linking similar levels of separate businesses. We refer to these relations by interconnectivity, composition, and collaboration. Underneath each horizontal relation's name, an example of conflict that could arise in a $\mathcal{B} 2 \mathcal{B}$ scenario is provided for illustration purposes. Collaboration relation bridges the strategic levels and focusses on how businesses adapt their goals and plans so that these businesses can now reach the goals that result out of their decision of partnership. Composition relation bridges the application levels and focusses on how new business processes are developed, either from scratch or after re-engineering existing processes. Finally, interconnectivity relation bridges the resource levels and focusses on the means that make the performance of business processes happen despite distribution and heterogeneity constraints.

\footnotetext{
${ }^{1}$ It is expected that joint decisions will be beneficial for all businesses, although personal interests might sometimes prevail, but this is outside this paper's scope.

${ }^{2}$ Decisions affecting a business growth are made at this level.
} 


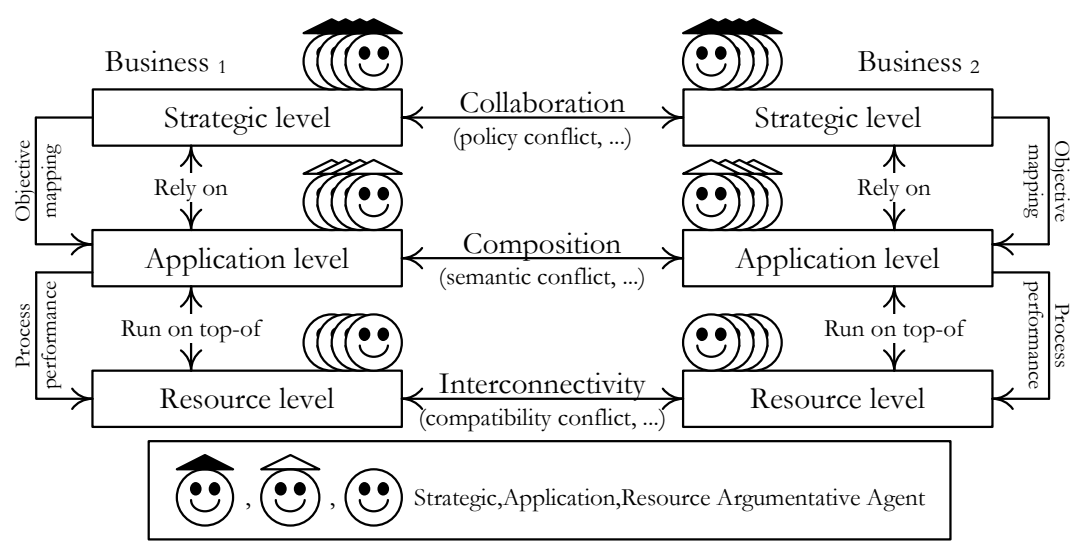

Figure 1. The argumentative agent framework for $\mathcal{B} 2 \mathcal{B}$ applications.

This paper is organized as follows. Section 2 introduces our agent-based framework for $\mathcal{B} 2 \mathcal{B}$ applications. Section 3 presents the argumentation model upon which this framework operates. Section 4 defines and discusses the argumentation-based protocol for $\mathcal{B} 2 \mathcal{B}$ conflict resolution and analyzes its formal and computational properties. To illustrate the proposed framework through a running example, an industrial case study (provided by IBM Research Division) is discussed in Section 5 along with its implementation using Java and logic programming with Prolog. Prior to concluding and presenting future work in Section 7, related work is discussed in Section 6.

\section{The Proposed Framework for $\mathcal{B} 2 \mathcal{B}$ Applications}

\subsection{Brief Description of Levels and Relations}

The resource level includes data and software resources (e.g., DBMS) that a business owns or manages, and the hardware resources upon which these software resources operate.

The application level is about the software applications that businesses operate such as payroll. From a $\mathcal{B} 2 \mathcal{B}$ perspective, the application level hosts a number of $\mathcal{A}$-AAs according to the number of these applications. The role of $\mathcal{A}$-AAs is to (i) monitor the external business processes that will use software applications and (ii) initiate interaction sessions with other $\mathcal{A}$-AAs. These sessions frame application compositions according to the guidelines that $\mathcal{S}$-AAs set and resolve possible conflicts during these compositions as depicted by composition relation in Fig. 1. For illustration purposes we assume that software applications are implemented as Web services [18], although other technologies could be used.

The strategic level is about the planning and decision-making mechanisms that underpin the growth of a business. Like the application level, the strategic level hosts a number of $\mathcal{S}$-AAs according to the number of active collaborations that a business initiates with its partners. The role of $\mathcal{S}$-AAs is to (i) reason over the business plans and (ii) initiate interaction sessions with other $\mathcal{S}$-AAs as depicted by collaboration relation 
in Fig. 1. These sessions aim at persuading peers to participate in collaborations, reviewing policies in case of conflicts, optimizing some parameters such as distribution network, etc. $\mathcal{A}$-AAs feed $\mathcal{S}$-AAs with details related to the execution progress of business processes. Particularly, if a conflict during the composition process cannot be resolved at the application level, the $\mathcal{A}$-AAs inform their respective $\mathcal{S}$-AAs.

From an argumentation perspective, the $\mathcal{S}$-AAs and $\mathcal{A}$-AAs are equipped with the same reasoning capabilities. However they differ in terms of the knowledge they manage and the responsibilities they are in charge of. For example, to resolve conflicts at the application or strategic levels, the $\mathcal{A}$-AAs or $\mathcal{S}$-AAs use the same persuasion and negotiation protocols but execute them differently. Protocols publicly describe the allowed moves, but how a certain move is selected would dependent on the knowledge that feed the agents' private strategies.

Fig. 1 shows vertical and horizontal relations. In a $\mathcal{B} 2 \mathcal{B}$ context, the focus is on horizontal relations. Interconnectivity relation targets the resource level and allows (i) data to freely and securely flow between businesses without any format, location, or semantic restriction and (ii) disparate resources to trigger each other without any access rights, time-slot availabilities, or compatibility restrictions. Communication protocols incompatibility (e.g., Synchronous Optical Networking (SONET) vs. Synchronous Digital Hierarchy (SDH)), incompatible hardware transmission technologies (e.g., circuit switching $v s$. multiplexing) and different modes of communication (e.g., connection-oriented communication vs. connectionless communication) are key examples of conflicts that fall under the interconnectivity relation.

Composition relation targets the application level and exhibits how business processes associated with $\mathcal{A}$-AAs get "virtually" integrated without being subject to any functional or structural changes. Lack of common semantics (e.g., different measurement units) is an example of conflict that falls under the composition relation. When it comes to Web services-based applications, composition targets users' requests that cannot be satisfied by any single, available Web service, whereas a composite Web service obtained by combining available Web services may be used.

Collaboration relation targets the strategic level and emphasizes the mechanisms that the $\mathcal{S}$-AAs set-up for coordinating the new $\mathcal{B} 2 \mathcal{B}$ processes using the $\mathcal{A}$-AAs. These processes are the result of composing applications, stretch beyond businesses' boundaries, and have to consider the requirements/limitations of the resource and application levels per business. For example, in the context of supply chain networks, many concrete conflicts that fall under the collaboration relation can arise. Concrete examples of such conflicts are: taxing policies incompatibility between manufactories and warehouses (e.g., various tax rates), incompatible scheduling policies between suppliers and manufactories (e.g., scheduling holidays during Christmas $v s$. summer) and incompatible inventory policies between distribution centers and retailers (e.g., lean $v s$. responsive inventory policies). Policies of businesses can be in contradiction, and some core business policies cannot be easily re-engineered. By using argumentative agents, we aim at handling these issues. Through their argumentative reasoning, and interaction, negotiation, and persuasion abilities, these agents could reason about these policies, identify possible conflicts, and update their policies to resolve these conflicts. They can, also, persuade each other for the benefit of collaborating and sharing their resources and determining alternative agents to work with, in case the current conflicts cannot be addressed. 


\subsection{Forms of Coordination}

Coordination, via explicit interactions between argumentative agents whether in the same or separate levels, is a pre-requisite to any successful $\mathcal{B} 2 \mathcal{B}$ application. We split coordination in the argumentative agent-based framework into two forms: vertical between strategic and application levels via the rely-on relation, and horizontal between strategic or application levels via the collaboration or composition relations, respectively. We discuss hereafter how argumentation is injected into coordination using Figs. 2 and 3 where plain lines and dotted lines denote interactions and conflict detection/resolution, respectively.

Vertical Coordination occurs within the boundaries of the same business. Here a $\mathcal{S}$-AA has the authority to execute a set of actions over an $\mathcal{A}$-AA (Fig. 2): "select", "ping", "trigger", "audit", and "retract \& select".

- "select" action makes the $\mathcal{S}$-AA identify the $\mathcal{A}$-AA of an application that will pursue the interactions with other $\mathcal{A}$-AAs as part of the partnership decision;

- "ping" action makes the $\mathcal{S}$-AA check the liveness and readiness of an application through its $\mathcal{A}$-AA; this is needed before this application is suggested as a potential candidate for receiving requests from other $\mathcal{A}$-AAs;

- "trigger" action makes the $\mathcal{S}$-AA forward the execution requests to the $\mathcal{A}$-AA of an application; these requests arrive from others $\mathcal{A}$-AAs;

- "audit" action makes the $\mathcal{S}$-AA monitor the performance of an application through its $\mathcal{A}$-AA; this is needed if the $\mathcal{S}$-AA has to guarantee a certain QoS to other $\mathcal{S}$-AAs.

- "retract \& select" make the $\mathcal{S}$-AA withdraw an application through its $\mathcal{A}$-AA based on the outcome of "audit" action (e.g., poor performance). This results in starting the search for another application to offer to other partners.

Argumentation in vertical coordination is illustrated with two cases: Application-toStrategic (this paper focus) and Strategic-to-Application.

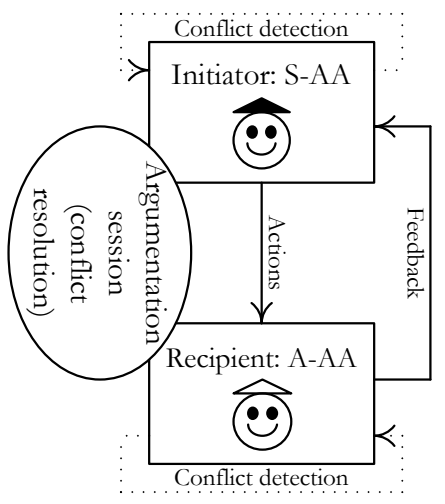

Figure 2. Argumentation in vertical coordination

Application-to-Strategic case highlights an $\mathcal{A}$-AA that faces difficulties in resolving conflicts and completing its operations. For example, the $\mathcal{A}$-AA was put on hold for a 
long period of time due to occupied resources or did not receive information in the right format from other businesses' $\mathcal{A}$-AAs. As a result, the $\mathcal{A}$-AA notifies its $\mathcal{S}$-AA so that both set-up an argumentation session for the sake of discussing the current difficulties and the potential solutions to put forward. This notification is represented with "feedback" in Fig. 2. Supply chain management is a concrete illustration of such a coordination. In this context, a conflict can happen between two partners about the delivery time. $\mathcal{S}$-AAs and $\mathcal{A}$-AAs of each player can argue to change the delivery time to satisfy the whole supply chain. Briefly, we report on the way conflict resolution progresses in this argumentation session.

1. Case 1. The $\mathcal{S}$-AA has an argument supporting the fact that the conflict facing the $\mathcal{A}$-AA could be resolved based on similar previous situations for example. Thus, the $\mathcal{S}$-AA argues with the $\mathcal{A}$-AA about the feasibility of this solution using persuasion (Section 4.2). If the $\mathcal{A}$-AA is not convinced (i.e., persuasion fails), the $\mathcal{S}$ AA will decide to select another $\mathcal{A}$-AA to continue the uncompleted composition work of the $\mathcal{A}$-AA that was withdrawn.

2. Case 2. The $\mathcal{S}$-AA does not have any argument for or against the possibility of resolving the conflicts that the $\mathcal{A}$-AA faces. Thus, the $\mathcal{S}$-AA and $\mathcal{A}$-AA collaborate to find a solution through an inquiry dialogue game like the one proposed in [7]. As defined by Walton and Krabbe [27], the inquiry dialogues rise from an initial situation of general ignorance and the purpose is to achieve the growth of knowledge and agreement.

3. If neither case $(\mathbf{1})$ or $(\mathbf{2})$ succeeds, the respective $\mathcal{S}$-AAs of the collaborative businesses try to work out a solution via horizontal coordination. When a solution is found, the $\mathcal{S}$-AAs check with the same $\mathcal{A}$-AAs if they are still available, or invite new $\mathcal{S}$-AAs to take part in the composition to deploy at the application level.

Strategic-to-Application case highlights a $\mathcal{S}$-AA that expects the occurrence of conflicts if appropriate actions are not taken on time. Examples of actions include reprimanding an $\mathcal{A}$-AA that released private details to peers. Expecting conflicts is based on the different feedbacks that the $\mathcal{S}$-AA receives from their $\mathcal{A}$-AAs. This shows a preventive strategy to conflict occurrence. However, handling preventive strategies is beyond the scope of this paper.

Horizontal Coordination spreads over the boundaries of businesses and thus, reflects $\mathcal{B} 2 \mathcal{B}$ applications in a better way. We identify two scenarios where each scenario involves either $\mathcal{S}$-AAs or $\mathcal{A}$-AAs. For the sake of simplicity, our description is restricted to $\mathcal{A}$-AAs. Here an $\mathcal{A}$-AA has the authority to carry out a set of actions over another peer engaged in the same composition (Fig. 3): "ping" and "trigger".

- "ping" action makes the $\mathcal{A}$-AA check the liveness of a remote application through its $\mathcal{A}$-AA; this is needed before the former $\mathcal{A}$-AA submits requests;

- "trigger" action makes the $\mathcal{A}$-AA submit its requests to a remote application through its $\mathcal{A}$-AA.

Argumentation in horizontal coordination is illustrated with two cases: Applicationto-Application and Strategic-to-Strategic.

Application-to-Application case stresses an $\mathcal{A}$-AA that identifies a conflict after interacting with peers. Conflicts could be of many types like different security policies, 


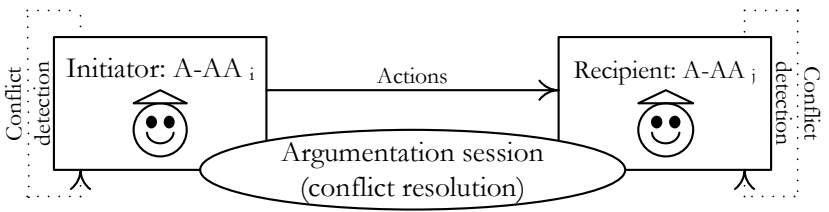

Figure 3. Argumentation in horizontal coordination

different semantics (e.g., different measurement units, different ontologies, etc.), conflicting quality of service, different costs associated with an application, etc.

$\mathcal{A}$-AAs try to resolve these conflicts via argumentation using a combination of persuasion and inquiry (Section 4.2). the $\mathcal{A}$-AA engage in pure persuasion if one of them has already a solution that could be accepted by the other with respect to the beliefs it has. However, merging persuasion with inquiry allows these agents to reach a joint argument.

Strategic-to-Strategic case highlights a $\mathcal{S}$-AA that identifies a conflict and tries to resolve it with its $\mathcal{S}$-AA partner. Some conflicts at this level concern penalty policies (e.g., collaboration's contract terms and conditions not respected) and payment policies. This case also stresses the situation where two $\mathcal{S}$-AAs of the collaborative businesses try to work out a solution of a conflict reported by the respective $\mathcal{A}$-AAs. This conflict cannot be resolved by vertical coordination. To this end, the $\mathcal{S}$-AAs engage in persuasion and inquiry sessions (Section 4.2).

Before detailing the protocol for persuasion and inquiry, we discuss our argumentative agents framework for $\mathcal{B} 2 \mathcal{B}$ applications that is based on computational argumentation theory.

\section{Formal Argumentation Framework}

\subsection{Background}

This section discusses the argumentation system that frames the internal operations in our $\mathcal{B} 2 \mathcal{B}$ framework. This discussion includes the configuration featuring argumentative agents as well. We use an abstract formal language $\mathcal{L}$ to express agents' beliefs. Here abstract means that beliefs could be propositional formulas like in [20], Horn clauses like in [4], or a set of facts and rules like in [7] and [12]. The use of an abstract language would make our framework generic and capable to capture properties that other frameworks concentrate on. The set of well-formed formulas ( $w f f$ ) built from $\mathcal{L}$ is denoted by $\mathcal{W} \mathcal{F}$. Agents build arguments using their beliefs. The set $\operatorname{Arg}(\mathcal{L})$ contains all those arguments. Similarly to $[1,8,9]$, we abstractly define argumentation as a dialectical process that underpins the exchange of "for/against" arguments that lead to some conclusion. Since we use an abstract language, we are not interested in the internal form of an argument. Formally, we define our argumentation framework as follows:

Definition 1 (Argumentation Framework) An abstract argumentation framework is a pair $\langle A, \mathcal{A T}\rangle$, where $A \subseteq \operatorname{Arg}(\mathcal{L})$ and $\mathcal{A T} \subseteq A \times A$ is a binary relation over $A$ that is not necessarily symmetric. For two arguments $a$ and $b$, we use $\mathcal{A T}(a, b)$ instead of $\mathcal{A T} \in(a, b)$ to indicate that $a$ is an attack against $b$. 
For example, an argument may be defined as a deduction of a conclusion from a given set of rules, or as a pair $(H, h)$ where $h$ is a sentence in $\mathcal{W F}$ and $H$ a subset of a given knowledge base such that (i) $H \vdash h$, (ii) $H$ is consistent, and (iii) there is no subset of $H$ with properties (i) and (ii). Like in formal argumentation theory (see for example [2,22,23]), we do not consider in this paper fuzzy arguments that could have a subjective nature. This means, when an argument is represented during an interaction, the agents can check whether an argument os legal or not. For example, if arguments are deducted from a given set of rules, the interacting agents are supposed to share the same rules so they can check if the deductions are valid, and if arguments are pairs, they can check if the three aforementioned conditions (i, ii, and iii) are satisfied. Consequently, as commonly supposed in the argumentation theory, the agents share the same definition of attack relation. This means, an argument $a$ attacks another argument $b$ iff $\mathcal{A T}(a, b)$ holds for all the interacting agents. In fact, as conflicts between arguments might occur, we need to define what an acceptable argument is. Dealing with fuzzy arguments could make the framework stronger and more flexible. However, this will raise complicated issues on how the arguments can be commonly accepted. Considering such issues is out of scope of this paper, and one of the major plans for our future work. To define the notion of acceptable arguments, we first define the notions of "defense" and "admissible set of arguments" (from [9,10]):

Definition 2 (Defense) Let $A \subseteq A r g(\mathcal{L})$ be a set of arguments over the argumentation framework, and let $S \subseteq A$. An argument a is defended by $S$ iff $\forall b \in A$ if $\mathcal{A T}(b, a)$, then $\exists c \in S: \mathcal{A T}(c, b)$.

Definition 3 (Admissible Set) Let $A \subseteq \operatorname{Arg}(\mathcal{L})$ be a set of arguments over the argumentation framework. A set $S \subseteq$ A of arguments is admissible iff:

1) $\nexists a, b \in S$ such that $\mathcal{A} \mathcal{T}(a, b)$ and

2) $\forall a \in S$ a is defended by $S$.

In other words, a set of arguments is admissible iff it is conflict-free and can counterattack every attack.

Example 1 Let $A=\{a, b, c, d\}$ and $\mathcal{A T}$ defined as follows: $\mathcal{A T}(b, a), \mathcal{A T}(c, a)$, $\mathcal{A T}(d, b), \mathcal{A T}(d, c)$. The sets: $\emptyset,\{d\}$, and $\{a, d\}$ are all admissible. However, the sets $\{b\}$ and $\{d, c\}$ are not admissible.

Definition 4 (Characteristic Function) Let $A \subseteq \operatorname{Arg}(\mathcal{L})$ be a set of arguments and let $S$ be an admissible set of arguments over the argumentation framework. The characteristic function of the argumentation framework is:

$$
\begin{aligned}
& F: 2^{A} \rightarrow 2^{A} \\
& F(S)=\{a \mid \text { a is defended by } S\}
\end{aligned}
$$

The following proposition shows a property of the characteristic function $F$. The proof is straightforward from Definitions 3 and 4.

Proposition 1 For all admissible set of arguments $S, S \subseteq F(S)$.

Definition 5 (Extensions) Let $S$ be an admissible set of arguments, and let $F$ be the characteristic function of the argumentation framework. 
- $S$ is a complete extension $\left(S_{\text {co }}\right)$ iff $S=F(S)$.

- $S$ is the grounded extension $\left(S_{g r}\right)$ iff $S=F(S)$ and $S$ is minimal (w.r.t. setinclusion) (grounded extension corresponds to the least fixed point of $F$ ).

- $S$ is a preferred extension $\left(S_{p r}\right)$ iff $S=F(S)$ and $S$ is maximal (w.r.t. setinclusion).

Example 2 Let us consider the same argumentation framework as in Example 1. We have:

- $F(\emptyset)=\{d\}$, so the admissible set $\emptyset$ is not a complete extension.

- $F(\{d\})=\{a, d\}$, so the admissible set $\{d\}$ is not a complete extension.

- $F(\{a, d\})=\{a, d\}$, so the admissible set $\{a, d\}$ is a complete extension.

In this example, the only complete extension $\{a, d\}$ is the grounded extension and is also the only preferred extension.

Example 3 Let $A=\{a, b, c\}$ and $\mathcal{A} \mathcal{T}$ defined as follows: $\mathcal{A T}(a, b)$ and $\mathcal{A} \mathcal{T}(b, a)$. The sets: $\{c\},\{a, c\}$, and $\{b, c\}$ are the complete extensions of the argumentation framework. The minimal complete extension $\{c\}$ is the grounded extension, and the maximal complete extensions $\{a, c\}$ and $\{b, c\}$ are the preferred extensions.

According to Definition 5, an admissible set $S$ is a complete extension if and only if $S$ is a fixed point of the function $F$, which means that all arguments defended by $S$ are also in $S$. Also, the grounded extension is the least fixed point of $F$. Consequently, the grounded extension contains all the arguments that are not attacked (the arguments that are defended by the empty set: $F(\emptyset)$ ), all the arguments that are defended by these non-attacked arguments $F(F(\emptyset))=F^{2}(\emptyset)$, all the arguments that are defended by the defended arguments $\left(F^{3}(\emptyset)\right)$, and so on until a fixed point is achieved. The grounded extension corresponds to the intersection of all the complete extensions. Finally, a preferred extension is a maximal complete extension that cannot be augmented by adding other arguments while staying complete.

We have the following direct proposition:

Proposition 2 Let $\langle A, \mathcal{A T}\rangle$ be an argumentation framework. $\exists ! S_{g r}$ in $\langle A, \mathcal{A T}\rangle$.

In other words, there exists a single grounded extension for the abstract argumentation framework. We can now define what the acceptable arguments in our system are.

Definition 6 (Acceptable Arguments) Let $A \subseteq \operatorname{Arg}(\mathcal{L})$ be a set of arguments, and let $G=S_{g r}$. An argument a over $A$ is acceptable iff $a \in G$.

According to this acceptability semantics, which is based on the grounded extension, if we have two arguments $a$ and $b$ such that $\mathcal{A T}(a, b)$ and $\mathcal{A T}(b, a)$, then $a$ and $b$ are both non-acceptable. In a $\mathcal{B} 2 \mathcal{B}$ scenario, this can happen when two $\mathcal{A}$-AAs present two conflicting arguments about the type of security policies to use for the current transaction: a weak policy that is simple to implement and less expensive or a strong policy that is hard to implement and more expensive. This notion is important in $\mathcal{B} 2 \mathcal{B}$ applications since agents should agree on an acceptable opinion, which is supported by an acceptable argument when a conflict arises. However, during the argumentative conversation, agents could use non-acceptable arguments as an attempt to change the status of some argu- 
ments previously uttered by the addressee, from acceptable to non-acceptable. This idea of using non-acceptable arguments in the dispute does not exist in the persuasion and inquiry protocols in the literature. For this reason, we introduce two new types of arguments based on the preferred extensions to capture this notion. We call these arguments semi-acceptable and preferred semi-acceptable arguments.

Definition 7 ((Preferred) Semi-Acceptable Arguments) Let $G$ be the grounded extension in the argumentation framework, and let $E_{1}, \ldots, E_{n}$ be the preferred extensions in the same framework. An argument a is:

- Semi-acceptable iff $a \notin G$ and $\exists E_{i}, E_{j}$ with $(1 \leq i, j \leq n)$ such that $a \in$ $E_{i} \wedge a \notin E_{j}$.

- Preferred semi-acceptable iff $a \notin G$ and $\forall E_{i}(1 \leq i \leq n) a \in E_{i}$.

In other words, an argument is semi-acceptable iff it is not acceptable and belongs to some preferred extensions, but not to all of them. An argument is preferred semiacceptable iff it is not acceptable and belongs to all the preferred extensions. The preferred semi-acceptable arguments are stronger than the semi-acceptable, and the grounded arguments are the strongest arguments in this classification.

Example 4 Let $A=\{a, b, c, d\}$ and $\mathcal{A T}$ is defined as follows: $\mathcal{A T}(a, b), \mathcal{A T}(b, a)$, $\mathcal{A T}(a, c), \mathcal{A T}(b, c)$, and $\mathcal{A} \mathcal{T}(c, d)$.

- $\emptyset$ is the grounded extension in this argumentation framework.

- The argumentation framework has two preferred extensions: $\{a, d\}$ and $\{b, d\}$. The arguments $a$ and $b$ are then semi-acceptable, and the argument $d$ is preferred semi-acceptable.

A concrete scenario of this example in a $\mathcal{B} 2 \mathcal{B}$ setting would be as follows: Suppose a transaction $\operatorname{Tr}$ along with three possible security policies: $s_{1}, s_{2}$, and $s_{3}$. The four arguments $a, b, c$ and $d$ are as follows:

- $a: s_{1}$ is the most suitable policy for the transaction $T r$.

- $b$ : Alone, $s_{2}$ is not sufficient to secure the transaction Tr, but by combining it with $s_{3}$ it becomes the most suitable.

- $c: s_{2}$ is less expensive than $s_{1}$.

- $d: s_{1}$ is not expensive to implement, and is sufficient to secure the transaction Tr.

To a certain extent, the argument $d$ is stronger than $a$ and b because it is defended by these two arguments against the only attacker $c$, and $a$ and $b$ attack each other. From a chronological point of view, we can imagine the following scenario leading to build these four arguments at the application level of two businesses represented by $\mathcal{A}-A A_{1}$ and $\mathcal{A}-A A_{2}$, respectively. First, $\mathcal{A}-A A_{1}$ presents the argument d, then $\mathcal{A}-A A_{2}$ attacks by moving forward the argument $c$. $\mathcal{A}-A A_{1}$ replies by attacking $c$ using the argument $a$. At that stage, the arguments a and $d$ are grounded. $\mathcal{A}-A A_{2}$ tries then to degrade one of these two arguments by attacking a using $b . \mathcal{A}-A A_{2}$ is aware that by using $b$ to attack $a, b$ is at the same time attacked by a. The idea here is just to change the status of the argument presented by $\mathcal{A}-A A_{1}$ from acceptable to semi acceptable.

Proposition 3 Let $A \subseteq \operatorname{Arg}(\mathcal{L})$ be a set of arguments, and let $S D=\{a \in A \mid \forall b \in$ $A \mathcal{A T}(b, a) \Rightarrow \mathcal{A T}(a, b) \& \nexists c \in A: \mathcal{A T}(c, b)\} . \forall a \in S D, a$ is semi acceptable. 
In other words, the arguments defending themselves by only themselves against all the attackers are semi-acceptable.

Proof see Appendix.

Proposition 4 Complete extensions are not closed under intersection.

Proof see Appendix.

Definition 8 (Eliminated Arguments) Let $A \subseteq \operatorname{Arg}(\mathcal{L})$ be a set of arguments, $a \in A$ be an argument, and $E L$ be the set of eliminated arguments over the argumentation framework. Also, let $E_{1}, \ldots, E_{n}$ be the preferred extensions in the same framework. $a \in E L$ iff $a \notin E_{i}, \forall i \in[1, n]$.

In other words, an argument is eliminated iff it does not belong to any preferred extension in the argumentation framework. We have the following proposition:

Proposition 5 Let a be an argument in $A$, and $A C, P S$, and $A$ be respectively the sets of acceptable, preferred semi-acceptable, and semi-acceptable arguments over the argumentation framework. $a \in E L$ iff $a \notin A C \cup P S \cup S A$.

In other words, an argument is eliminated iff it is not acceptable, not preferred semiacceptable, and also not semi-acceptable.

Proof see Appendix.

Consequently, arguments take four exclusive statuses namely acceptable, preferred semi-acceptable, semi-acceptable, and eliminated. The dynamic nature of agent interactions is reflected by the changes in the statuses of the uttered arguments.

\subsection{Partial Arguments and Conflicts for $\mathcal{B} 2 \mathcal{B}$ Applications}

In a $\mathcal{B} 2 \mathcal{B}$ scenario, it happens that the argumentative agents $\mathcal{S}$-AAs and $\mathcal{A}$-AAs do not have complete information about some facts. In a similar situation, they can build partial arguments for some conclusions out of their beliefs. We define a partial argument as follows:

Definition 9 (Partial Arguments) Let $x$ be a wff in $\mathcal{W F}$. A partial argument denoted by $a_{x}^{p}$ is part of an argument $a \in A$, which misses an argument (or a proof) for $x$. In other words, by adding a proof supporting $x$ to $a_{x}^{p}$ an argument is obtained.

For example, if arguments are defined as deductions from a set of rules, $x$ will represent some missing rules, and if arguments are defined as pairs $(H, h), x$ will represent a subset of $H$.

Example 5 let us suppose that arguments are defined as pairs $(H, h)$ using propositional logic. $a=((m, m \rightarrow n), n)$ is an argument for $n$ and $a_{x}^{p}=((m \rightarrow n), n)$ is a partial argument for $n$ missing the support for $x=m$.

$a=((m, m \rightarrow n, n \rightarrow l, l \rightarrow r), r)$ is an argument for $r$ and $a_{x}^{p}=((n \rightarrow l, l \rightarrow r), r)$ is a partial argument for $r$ missing the support for $x=n$. In this case, a possible support is $((m, m \rightarrow n), n)$. 
In a $\mathcal{B} 2 \mathcal{B}$ scenario, an example where partial arguments are needed is when $\mathcal{A}$ $\mathrm{AA}_{1}$ of business $B_{1}$ knows that security policy $s_{2}$ that another business $B_{2}$ uses can be substituted by policy $s_{1}$ that $B_{1}$ uses if some conditions are met when deploying $s_{2}$. Thus, $\mathcal{A}-\mathrm{AA}_{1}$ can build a partial argument supporting the fact that $B_{2}$ can use $s_{1}$. To be an argument, this partial argument needs a support that implementing $s_{2}$ in $B_{2}$ meets these conditions.

The idea behind building partial arguments by an agent is to check if the other agent can provide the missing part or a part of this missing part so that the complete argument could be jointly built (progressively). This idea which is a part of the inquiry dialogue will be made clear in the persuasion protocol defined in Section 4.2.

As for arguments, we need to define what an acceptable partial argument is. This acceptability is defined in the same way as for arguments. We use the notation $a_{x}^{p} . x$ to denote the resulting argument of combining the partial argument $a_{x}^{p}$ and an argument supporting $x$ supposing that this latter exists.

Definition 10 (Partial Attack) Let $a_{x}^{p}$ be a partial argument over the argumentation framework. $\mathcal{A T}\left(a_{x}^{p}, b\right)$ iff $\mathcal{A} \mathcal{T}\left(a_{x}^{p} . x, b\right)$ and $\mathcal{A} \mathcal{T}\left(b, a_{x}^{p}\right)$ iff $\mathcal{A} \mathcal{T}\left(b, a_{x}^{p} . x\right)$.

Definition 11 (Acceptable Partial Arguments) A partial argument $a_{x}^{p}$ is acceptable (preferred semi-acceptable, semi-acceptable) iff $a_{x}^{p} . x$ is acceptable (preferred semiacceptable, semi-acceptable).

Example 6 Let $\Sigma=\{n \rightarrow m, r \rightarrow l, l \rightarrow t, \neg l\}$ be a propositional knowledge base. The partial argument $((n \rightarrow m), m)$ is acceptable, however the partial argument $((r \rightarrow$ $l, l \rightarrow t), t)$ is not acceptable since the argument $((r, r \rightarrow l, l \rightarrow t), t)$ is attacked by the argument $(\neg l, \neg l)$.

Proposition 6 Let $a$ be an argument in A. If a is acceptable, then $\forall x \in \mathcal{W F} a_{x}^{p}$ is acceptable.

Proof see Appendix.

After specifying the argumentation model, We define the notions of conflict and conflict resolution in our $\mathcal{B} 2 \mathcal{B}$ framework as follows:

Definition 12 (Conflict) Let $p$ and $q$ be two wffs in $\mathcal{W F}$. There is a conflict between two argumentative agents $\alpha$ and $\beta$ about $p$ and $q$ in the $\mathcal{B} 2 \mathcal{B}$ framework iff one of them (e.g., $\alpha$ ) has an acceptable argument a for $p$ (denoted $a \uparrow p$ ) and the other (i.e., $\beta$ ) has an acceptable argument b for $q(b \uparrow q)$ such that $\mathcal{A T}(a, b)$ or $\mathcal{A T}(b, a)$. We denote this conflict by $\alpha_{p} ¥ \beta_{q}$.

For example, if $p$ and $q$ represent each a security policy $s_{1}$ and $s_{2}$ such that $s_{1}$ and $s_{2}$ cannot be used together, then there is a conflict if one agent has an acceptable argument for using $s_{1}$ while the other agent has an acceptable argument for using $s_{2}$ (the two arguments are conflicting). This conflict arises when both agents need to agree on which security policy to use.

Before defining the notion of conflict resolution, we need to define the notions of interaction and outcome of interaction. An utterance $u$ made by an agent $\alpha$ in a given interaction is denoted $u \rightsquigarrow \alpha$. 
Definition 13 (Interaction) Let $\alpha$ and $\beta$ be two argumentative agents. An interaction (denoted by $I_{\alpha, \beta}$ ) between $\alpha$ and $\beta$ in the $\mathcal{B} 2 \mathcal{B}$ framework is an ordering sequence of utterances $u_{1}, u_{2}, \ldots, u_{n}$ such that $u_{i} \rightsquigarrow \alpha \Rightarrow u_{i+1} \rightsquigarrow \beta$ and $u_{i} \rightsquigarrow \beta \Rightarrow u_{i+1} \rightsquigarrow \alpha$. $C S_{\alpha}$ (resp. $\left.C S_{\beta}\right)$ is the set (called commitment store) containing the arguments used by $\alpha$ (resp. $\beta)$ during the interaction.

Definition 14 (Conflict Resolution) Let $p$ and $q$ be two wffs in $\mathcal{W F}$ and $\alpha$ and $\beta$ be two argumentative agents in the $\mathcal{B} 2 \mathcal{B}$ framework such that $\alpha_{p} ¥ \beta_{q}$. Also let $I_{\alpha, \beta}$ be an interaction in this framework. The conflict $\alpha_{p} ¥ \beta_{q}$ is resolved by the interaction $I_{\alpha, \beta}$ iff the outcome of $I_{\alpha, \beta}$ is a formula $r \in \mathcal{W F}$ such that $\exists a \in C S_{\alpha}, b \in C S_{\beta}: a \uparrow r, b \uparrow r$ and $a$ and $b$ are both acceptable.

In the aforementioned security example, the conflict is resolved iff (i) after interaction, one of the agents can build an acceptable argument from its knowledge base and the arguments exchanged during this interaction, supporting the use of the other agent's policy, or (ii) when both agents agree on the use of a new policy such that each agent can build an acceptable argument, from its knowledge base and the exchanged arguments, supporting the use of this policy. The idea here is that by exchanging arguments, new solutions (and arguments supporting these solutions) can emerge. In this case, the agents should update their beliefs by withdrawing attacked (i.e., eliminated) assumptions. However, there is still a possibility that each agent keeps its own viewpoint at the end of the conversation.

\section{Argumentative Persuasion for $\mathcal{B} 2 \mathcal{B}$}

\subsection{Notations}

The outcome of an interaction to resolve a conflict in a $\mathcal{B} 2 \mathcal{B}$ setting depends on the status of the formula representing the conflict topic. As for arguments, a wff has four statuses depending on the statuses of the arguments supporting it (an argument supports a formula if this formula is the conclusion of that argument). A wff is acceptable if there exists an acceptable argument supporting it. If not, and if there exists a preferred semi-acceptable argument supporting it, then the formula is preferred semi-acceptable. Otherwise, the formula is semi-acceptable if a semi-acceptable argument supporting it exists, or eliminated if such an argument does not exist. Let $S t$ be the set of these statuses. We define the following function that returns the status of a $w f f$ with respect to a set of arguments:

$$
\Delta: \mathcal{W F} \times 2^{A} \rightarrow S t
$$

Generally, the interactions that are needed in a $\mathcal{B} 2 \mathcal{B}$ scenario involve two argumentative agents. For simplicity, we will not refer in the remainder of the paper to agent types (strategic or application), but denote participating agents by $\alpha$ and $\beta$. Each agent has a possibly inconsistent belief base $\Sigma_{\alpha}$ and $\Sigma_{\beta}$, respectively, that contains for example, all the policies upon which these agents should reason when they manage businesses as explained in the previous sections. Although the argumentation framework presented in Section 3 does not assume that arguments are truthful, we suppose that the agents partic- 
ipating in resolving $\mathcal{B} 2 \mathcal{B}$ conflicts are truth telling. This means they (i) reveal only the arguments they have in their knowledge bases and (ii) are perfectly cooperative. Promoting truth telling in the proposed framework using for instance game-theoretical techniques, and considering non-cooperative agents are plans for future work.

Agents use their argumentation systems to decide about the next move to play (e.g., accept or attack the arguments put forward during their interactions). When an agent accepts an argument that an addressee suggests, this agent updates its knowledge base by adding the elements of this argument and removing all the elements that attack this argument. The acceptability we consider here is purely argumentative as specified in Definition 6. However, if businesses have different power positions in their relationship, the proposed approach still stands if the power positions are clearly stated as shared and agreed upon rules. This simply means that acceptability should also consider these rules. For example, an agent can accept an argument if it is associated with a power. Furthermore, an argument $a$ can attack another argument $b$ without being attacked by this argument (i.e., by $b$ ) if $a$ is given by an agent having a superior power position. However, if these rules are not shared and agreed upon, they can still be used simply by not being cooperative, which is an assumption for the soundness of our approach.

Each agent $\alpha$ has also a commitment store $\left(C S_{\alpha}\right)$ that is publicly accessible for consultation but only updated by the owner agent. The commitment stores are empty when interaction starts, and updated by adding arguments and partial arguments that the agents exchange. $C S_{\alpha}$ refers to the commitment store of agent $\alpha$ at the current moment.

The possibility for an agent $\alpha$ to build an acceptable argument $a$ (respectively an acceptable partial argument $a_{x}^{p}$ ) from its knowledge base and the commitment store of the addressee $\beta$ is denoted by $\mathcal{A R}\left(\Sigma_{\alpha} \cup C S_{\beta}\right) \triangleright a$ (respectively $\left.\mathcal{A R}\left(\Sigma_{\alpha} \cup C S_{\beta}\right) \triangleright a_{x}^{p}\right)$. Building a partial argument $a_{x}^{p}$ from a knowledge base means that no argument for or against $x$ can be built. $\mathcal{A R}\left(\Sigma_{\alpha} \cup C S_{\beta}\right) \not a$ (respectively $\left.\mathcal{A R}\left(\Sigma_{\alpha} \cup C S_{\beta}\right) \not a_{x}^{p}\right)$ means that agent $\alpha$ cannot build an acceptable argument $a$ (respectively an acceptable partial argument $a_{x}^{p}$ ) from $\Sigma_{\alpha} \cup C S_{\beta}$. The symbols $\unrhd$ and $\unrhd$ associated with the semi-acceptable (partial) arguments are defined in the same way.

\subsection{Protocol Specification}

In our $\mathcal{B} 2 \mathcal{B}$ framework, the agents engage in persuasion and inquiry dialogues to resolve conflicts. Atkinson et al. [2], Pasquier et al. [21], and Prakken [22] propose persuasion protocols for multi-agent systems. However, these protocols consider only pure persuasion without inquiry stages and do not address completeness (or pre-determinism) property [20]. We propose a persuasion protocol that includes inquiry stages in our $\mathcal{B} 2 \mathcal{B}$ framework, in which pre-determinism is considered. The protocol is modeled with dialogue games $[16,17]$. Dialogue games are interactions between players (agents), in which each player moves by performing utterances according to a pre-defined set of rules. Let us define the notions of protocol and dialogue games.

Definition 15 (Protocol) A protocol is a pair $\langle\mathcal{C}, \mathcal{D}\rangle$ with $\mathcal{C}$ a finite set of allowed moves and $\mathcal{D}$ a set of dialogue games.

The moves in $\mathcal{C}$ are of $c$ different types $(c>0)$. We denote by $M_{i}(\alpha, \beta, a, t)$ a move of type $i$ played by agent $\alpha$ and targeting agent $\beta$ at time $t$ regarding a content $a$. We consider four types of moves in our protocol: Assert, Accept, Attack, and Question. Gen- 
erally, in the persuasion protocol the agents exchange arguments. Except the Question move whose content is not an argument, the content of other moves is an argument $a$ $(a \in \operatorname{Arg}(\mathcal{L}))$. When replying to a Question move, the content of Assert move can also be a partial argument or "?" when the agent does not know the answer. We use another particular move Stop with no content. It could be used by an agent to stop the interaction.

Intuitively, a dialogue game in $\mathcal{D}$ is a rule indicating the possible moves that an agent could play following a move done by an addressee. This is specified formally as follows:

Definition 16 (Dialogue Game) A dialogue game $\mathrm{Dg}$ is either of the form:

$$
M_{i}\left(\alpha, \beta, a_{i}, t\right) \Rightarrow \bigvee_{0<j \leq n_{i}} M_{j}\left(\beta, \alpha, a_{j}, t^{\prime}\right)
$$

where $M_{i}$ and $M_{j}$ are in $\mathcal{C}, t<t^{\prime}$ and $n_{i}$ is the number of allowed moves that $\beta$ could perform after receiving a move of type i from $\alpha$;

or of the form:

$$
\Rightarrow \bigvee_{0<j \leq n} M_{j}\left(\alpha, \beta, a_{j}, t_{0}\right)
$$

where $M_{j}$ are in $\mathcal{C}, t_{0}$ is some initial time, and $n$ is the number of allowed moves that $\alpha$ could perform initially.

According to this definition, a dialogue game could be either deterministic (if $n=1$ and $\forall i n_{i}=1$ ) or non-deterministic. Because agents are autonomous, we consider here non-deterministic dialogue games, in that, for example, given an incoming move of type $i$, the receiving agent needs to choose amongst the $n_{i}$ possible replies $\left(n_{i}>1\right)$. As proposed in $[4,6,7]$, we combine public dialogue games with private strategies so that the agents become deterministic. To this end we introduce the conditions within dialogue games, each associated with a single reply.

Definition 17 (Strategic Dialogue Game) A strategic dialogue game $S D g$ is a conjunction of rules, specified either as follows:

$$
\bigwedge_{0<j \leq n_{i}}\left(M_{i}(\alpha, \beta, a, t) \wedge C_{j} \Rightarrow M_{j}\left(\beta, \alpha, a_{j}, t^{\prime}\right)\right)
$$

where $t<t^{\prime}$ and $n_{i}$ is the number of allowed communicative acts that $\beta$ could perform after receiving a move of type i from $\alpha$;

or as follows:

$$
\bigwedge_{0<j \leq n}\left(C_{j} \Rightarrow M_{j}\left(\alpha, \beta, a_{j}, t_{0}\right)\right)
$$

where $t_{0}$ is the initial time and $n$ is the number of allowed moves that $\alpha$ could play initially. 
In order to guarantee determinism, conditions $C_{j}$ need to be mutually exclusive [25]. The agents use their argumentation systems to evaluate, in a private manner, conditions $C_{j}$. These argumentation systems are based on the private agents' beliefs and the public commitments recorded in the commitment stores.

To simplify the notations, we omit the time parameter form the moves and use the notation $\cup C S$ as an abbreviation of $C S_{\alpha} \cup C S_{\beta}$. In our $\mathcal{B}$ usiness-to- $\mathcal{B}$ usiness $\mathcal{P}$ ersuasive $\mathcal{P}$ rotocol $(\mathcal{B} 2 \mathcal{B}-\mathcal{P} \mathcal{P})$, the agents are not allowed to play the same move (with the same content) more than once. The strategic dialogue games we consider in this protocol are:

\section{1- Initial game}

$$
C_{i n 1} \Rightarrow \operatorname{Assert}(\alpha, \beta, a)
$$

where:

$$
C_{i n 1}=\exists p, q \in \mathcal{W F}: \alpha_{p} ¥ \beta_{q} \wedge \mathcal{A R}\left(\Sigma_{\alpha}\right) \triangleright a \wedge a \uparrow p
$$

Although generic, this game is derived from $\mathcal{B} 2 \mathcal{B}$ settings where a business detects a conflict with another business. The persuasion starts when a conflict is detected and one of the two businesses/agents asserts an acceptable argument that supports its position. In the remainder of this section, we suppose that the persuasion topic is represented by the $w f f p$.

\section{2- Assertion game}

$$
\begin{aligned}
& A \operatorname{sert}(\alpha, \beta, \mu) \wedge C_{a s 1} \Rightarrow \operatorname{Attack}(\beta, \alpha, b) \\
& A \operatorname{sert}(\alpha, \beta, \nu) \wedge C_{a s 2} \Rightarrow \operatorname{Question}(\beta, \alpha, x) \wedge \\
& A \operatorname{ssert}(\alpha, \beta, \nu) \wedge C_{a s 3} \Rightarrow \operatorname{Accept}(\beta, \alpha, a) \\
& A \operatorname{siert}(\alpha, \beta, \nu) \wedge C_{a s 4} \Rightarrow \operatorname{Stop}(\beta, \alpha)
\end{aligned}
$$

where $\mu$ is an argument or partial argument, $\nu$ is an argument, partial argument, or "?" and:

$$
\begin{aligned}
C_{a s 1}= & O p_{a s_{1}}^{a t_{1}} \vee\left(\neg O p_{a s_{1}}^{a t_{1}} \wedge O p_{a s_{1}}^{a t_{2}}\right) \\
O p_{a s_{1}}^{a t_{1}}= & \exists b \in A \mathcal{A}\left(\Sigma_{\beta} \cup C S_{\alpha}\right) \triangleright b \\
& \wedge \Delta(p, \cup C S) \neq \Delta(p, \cup C S \cup\{b\}) \\
O p_{a s_{1}}^{a t_{2}}= & \exists b \in A: \mathcal{A R}\left(\Sigma_{\beta} \cup C S_{\alpha}\right) \unrhd b \\
& \wedge \Delta(p, \cup C S) \neq \Delta(p, \cup C S \cup\{b\}) \\
C_{a s 2}= & \neg C_{a s 1} \wedge\left(O p_{a s_{2}}^{q u_{1}} \vee\left(\neg O p_{a s_{2}}^{q u_{1}} \wedge O p_{a s_{2}}^{q u_{2}}\right)\right) \\
O p_{a s_{2}}^{q u_{1}}= & \exists b_{x}^{p}, b_{x}^{p} \cdot x \in A \mathcal{A R}\left(\Sigma_{\beta} \cup C S_{\alpha}\right) \triangleright b_{x}^{p} \\
& \wedge \Delta(p, \cup C S) \neq \Delta\left(p, \cup C S \cup\left\{b_{x}^{p} . x\right\}\right) \\
O p_{a s_{2}}^{q u_{2}}= & \exists b_{x}^{p}, b_{x}^{p} \cdot x \in A: \mathcal{A R}\left(\Sigma_{\beta} \cup C S_{\alpha}\right) \unrhd b_{x}^{p} \\
& \wedge \Delta(p, \cup C S) \neq \Delta\left(p, \cup C S \cup\left\{b_{x}^{p} \cdot x\right\}\right) \\
\left.C_{a s 3} \cdot x\right\} & \exists a \in A: \mathcal{A R}\left(\Sigma_{\beta} \cup C S_{\alpha}\right) \triangleright a \wedge a \uparrow p \\
C_{a s 4}= & \neg O p_{a s_{1}}^{a t_{1}} \wedge \neg O p_{a s_{2}}^{q u_{1}} \wedge \neg O p_{a s_{2}}^{q u_{2}} \wedge \neg C_{a s 3} \\
& \wedge \forall b \in A, \mathcal{A R}\left(\Sigma_{\beta} \cup C S_{\alpha}\right) \unrhd b \Rightarrow \\
& \Delta(p, \cup C S)=\Delta(p, \cup C S \cup\{b\})
\end{aligned}
$$


In this game, the content of Assert could be an argument, partial argument, or "?". The last two choices are particularly derived from $\mathcal{B} 2 \mathcal{B}$ aplications, where businesses have only partial information about each other's activities and should exchange questions to reveal the unkown information that could help in resolving the business conflict. We recall that the two businesses are cooperative because they have interest in resolving the conflict. Indeed, businesses/agents can use this move to assert new arguments in the initial game or to reply to a question in the question game, which is a part of inquiry in our protocol. The move that agent $\beta$ can play as a reply to the Assert move depends on the content of this assertion. When $\alpha$ asserts an argument or a partial argument, $C S_{\alpha}$ changes by adding the advanced (partial) argument. Agent $\beta$ can attack agent $\alpha$ if $\beta$ can generate an acceptable argument from its knowledge base and the $\alpha$ 's commitment store so that this argument will change the status of the persuasion topic. Consequently, in this protocol the agents do not attack only the recently advanced argument, but any advanced argument during the interaction, which is still acceptable or (preferred) semi-acceptable $\left(O p_{a s_{1}}^{a t_{1}}\right)$. This makes the protocol more flexible and efficient (for example agents can try different arguments to attack a given argument). If such an acceptable argument cannot be generated, $\beta$ will try to generate a (preferred) semi-acceptable argument changing the status of $p\left(O p_{a s_{1}}^{a t_{2}}\right)$. The idea here is that if $\beta$ eliminates $\alpha$ 's arguments, it will try to make them (preferred) semi-acceptable. This is due to the following proposition whose proof is straightforward from the definition of semi-acceptable arguments and the fact that only four statuses are possible.

Proposition 7 If $\beta$ plays the Attack move with a semi-acceptable argument, then the $\alpha$ 's attacked argument changes the status from acceptable to semi-acceptable, and the persuasion topic changes the status from acceptable to semi-acceptable or preferred semi-acceptable.

We notice that in the Assertion game changing the status of $p$ is a result of an attack relation:

Proposition 8 In the Assertion game we have: $\forall b \in A$, $\Delta(p, \cup C S) \neq \Delta(p, \cup C S \cup\{b\}) \Rightarrow \exists a \in \cup C S: \mathcal{A T}(b, a)$.

If $\beta$ cannot play the Attack move, then before checking the acceptance of an $\alpha$ 's argument, it checks if no acceptable and then no (preferred) semi-acceptable argument in the union of the knowledge bases can attack this argument (inquiry part). For that, if $\beta$ can generate a partial argument changing the status of $p$, then it will question $\alpha$ about the missing assumptions $\left(O p_{a s_{2}}^{q u_{1}}\right.$ and $\left.O p_{a s_{2}}^{q u_{2}}\right)$. This new feature provides a solution to the "pre-determinism" problem identified in [20]. If such a partial argument does not exist, and if $\beta$ can generate an acceptable argument supporting $p$, then it plays the Accept move $\left(C_{a s 3}\right)$.

Proposition 9 An agent plays the Accept move only if it cannot play the Attack move and cannot play the Question move.

Proof see Appendix.

Agent $\beta$ plays the Stop move when it cannot accept an $\alpha$ 's argument and cannot attack it. This happens when an agent has a semi-acceptable argument for $p$ and another semi- 
acceptable argument against $p$, so the status of $p$ in the union of the commitment stores will not change by advancing the $\beta$ 's argument $\left(C_{a s 4}\right)$. Finally, we notice that if the content of Assert move is "?", $\beta$ cannot play the Attack move. The reason is that such an Assert is played after a question in the Question game, and the agents play Question moves only if an attack is not possible. Using simple logical calculus, we can prove the following proposition:

Proposition 10 An agent plays the Stop move iff it cannot play another move.

\section{3- Attack game}

$$
\begin{aligned}
& \operatorname{Attack}(\alpha, \beta, a) \wedge C_{a t 1} \Rightarrow \operatorname{Attack}(\beta, \alpha, b) \\
& \operatorname{Attack}(\alpha, \beta, a) \wedge C_{a t 2} \Rightarrow \operatorname{Question}(\beta, \alpha, x) \\
& \operatorname{Attack}(\alpha, \beta, a) \wedge C_{a t 3} \Rightarrow \operatorname{Accept}(\beta, \alpha, a) \\
& \operatorname{Attack}(\alpha, \beta, a) \wedge C_{a t 4} \Rightarrow \operatorname{Stop}(\beta, \alpha)
\end{aligned}
$$

where:

$$
\begin{aligned}
C_{a t 1}= & O p_{a t_{1}}^{a t_{1}} \vee\left(\neg O p_{a t_{1}}^{a t_{1}} \wedge O p_{a t_{1}}^{a t_{2}}\right) \\
O p_{a t_{1}}^{a t_{1}}= & O p_{a s_{1}}^{a t_{1}} \\
O p_{a t_{1}}^{a t_{2}}= & O p_{a s_{1}}^{a t_{1}} \\
C_{a t 2}= & \neg C_{a t_{1}} \wedge\left(O p_{a t_{2}}^{q u_{1}} \vee\left(\neg O p_{a t_{2}}^{q u_{1}} \wedge O p_{a t_{2}}^{q u_{2}}\right)\right) \\
O p_{a t_{2}}^{q u_{1}}= & O p_{a s_{2}}^{q u_{1}} \\
O p_{a t_{2}}^{q u_{2}}= & O p_{a s_{2}}^{q u_{2}} \\
C_{a t 3}= & \mathcal{A R}\left(\Sigma_{\beta} \cup C S_{\alpha}\right) \triangleright a \wedge \neg O p_{a t_{2}}^{q u_{1}} \wedge \neg O p_{a t_{2}}^{q u_{2}} \\
C_{a t 4}= & \neg O p_{a t_{1}}^{a t_{1}} \wedge \neg O p_{a t_{2}}^{q u_{1}} \wedge \neg O p_{a t_{2}}^{q u_{2}} \wedge \neg C_{a t 3} \\
& \wedge \forall b \in A, \mathcal{A R}\left(\Sigma_{\beta} \cup C S_{\alpha} \unrhd b \Rightarrow\right. \\
& \Delta(p, \cup C S)=\Delta(p, \cup C S \cup\{b\})
\end{aligned}
$$

The conditions associated with the Attack game are similar to the ones defining the Assert game. The Attack move also includes the case where the business/agent that initiates the persuasion puts forward a new argument, which is not attacking any existing argument but changing the status of the persuasion topic. This is particularly useful in $\mathcal{B} 2 \mathcal{B}$ applications when the advanced arguments cannot be attacked/defended, so that the business conflict cannot be resolved. However, by trying another way to convince the addressee, the initiator business/agent can achieve the purpose of resolving successfully the conflict.

\section{4- Question game}

$$
\begin{aligned}
& \text { Question }(\alpha, \beta, x) \wedge C_{q u 1} \Rightarrow A \operatorname{ssert}(\beta, \alpha, a) \wedge \\
& \text { Question }(\alpha, \beta, x) \wedge C_{q u 2} \Rightarrow A \operatorname{ssert}\left(\beta, \alpha, y_{x^{\prime}}^{p}\right) \wedge \\
& \text { Question }(\alpha, \beta, x) \wedge C_{q u 3} \Rightarrow A \operatorname{ssert}(\beta, \alpha, ?)
\end{aligned}
$$

where: 


$$
\begin{aligned}
C_{q u 1}= & \exists a \in A: \mathcal{A R}\left(\Sigma_{\beta} \cup C S_{\alpha}\right) \triangleright a \wedge(a \uparrow x \vee a \uparrow \bar{x}) \\
C_{q u 2}= & \exists y_{x^{\prime}}^{p}, y_{x^{\prime}}^{p} x^{\prime} \in A: \mathcal{A R}\left(\Sigma_{\beta} \cup C S_{\alpha}\right) \triangleright y_{x^{\prime}}^{p} \\
& \wedge\left(y_{x^{\prime}}^{p} \uparrow x \vee y_{x^{\prime}}^{p} \uparrow \bar{x}\right) \\
C_{q u 23}= & \neg C_{q u 1} \wedge \neg C_{q u 2}
\end{aligned}
$$

Business/agent $\beta$ can answer the $\alpha$ 's question about the content $x$ by asserting an argument for or against $x$. If not, it answers by a partial argument if it can generate it. Otherwise, it answers by "?", which means that it does not know if $x$ holds or not. This game is particularly derived from $\mathcal{B} 2 \mathcal{B}$ applications where the information about the involved businesses are limited. The game is played when a business/agent has a partial argument and asks the other business/agent about the missing part that is needed to achieve an acceptable resolution of the conflict, so that the answer could be the complete missing part, a part of it, or nothing.

\section{5- Stop game}

$$
\begin{aligned}
& \operatorname{Stop}(\alpha, \beta) \wedge C_{s t 1} \Rightarrow \text { Question }(\beta, \alpha, x) \wedge \\
& \operatorname{Stop}(\alpha, \beta) \wedge C_{\text {st } 2} \Rightarrow \operatorname{Stop}(\beta, \alpha)
\end{aligned}
$$

where:

$$
\begin{array}{ll}
C_{s t 1} & \left.=O p_{s t 1_{1}}^{q u_{1}} \vee\left(\neg O p_{s t_{1}}^{q u_{1}} \wedge O p_{s t_{1}}^{q u_{2}}\right)\right) \\
O p_{s t_{1}}^{q u_{1}} & =O p_{a s_{2}}^{q u_{1}} \\
O p_{s 1_{1}}^{q u_{2}} & =O p_{a s_{2}}^{q u_{2}} \\
C_{s t 2} & =\neg C_{s t 1}
\end{array}
$$

Before answering the $\alpha$ 's Stop move by another Stop to terminate the persuasion, $\beta$ checks if no other partial arguments changing the status of $p$ could be generated. Consequently, the Stop move is played only if no such argument could be generated, which means that the conflict cannot be resolved. As mentioned above, checking the existence of other partial arguments is particularly useful for $\mathcal{B} 2 \mathcal{B}$ applications as the shared information are usually partial and incomplete.

\subsection{Protocol Analysis}

In this section, we prove the termination, soundness, and completeness of $\mathcal{B} 2 \mathcal{B}-\mathcal{P} \mathcal{P}$ and discuss its computational complexity.

Theorem $1 \mathcal{B} 2 \mathcal{B}-\mathcal{P} \mathcal{P}$ always terminates either successfully by Accept or unsuccessfully by Stop.

Proof see Appendix.

When the protocol terminates, we define its soundness and completeness as follows:

Definition 18 (Soundness - Completeness) A persuasion protocol about a wff $p$ is sound and complete iff for some arguments a for or against $p$ we have:

$\mathcal{A R}\left(\Sigma_{\alpha} \cup \Sigma_{\beta}\right) \triangleright a \Leftrightarrow \mathcal{A R}(\cup C S) \triangleright a$. 
Theorem 2 The protocol $\mathcal{B} 2 \mathcal{B}-\mathcal{P} \mathcal{P}$ is sound and complete.

Proof see Appendix.

As discussed earlier, the agents are supposed to be truth telling and cooperative. The following theorem gives the result if such an assumption is not considered.

Theorem 3 The soundness and completeness of the protocol $\mathcal{B} 2 \mathcal{B}-\mathcal{P} \mathcal{P}$ do not hold if agents are not truth telling.

Proof see Appendix.

This means if the agents are not truth telling, the interaction outcome cannot be determined, as it could be anything.

The computational complexity of the protocol can be computed considering two issues: 1) the complexity of computing arguments, partial arguments, and their acceptability; and 2) the complexity of playing the games. An important issue in this complexity is the underlying logical language used to specify arguments. It has been proved in [4] that when Horn clauses are used, the complexity of computing arguments and their acceptability is polynomial if the knowledge base is consistent or is under the form of definite Horn. Because partial arguments are also arguments that miss given parts, the complexity of their computation along with their acceptability is also polynomial in the case of Horn clauses. We notice that for the purpose of $\mathcal{B} 2 \mathcal{B}$ applications, Horn clauses, which are disjunction of literals (an elementary or atomic proposition or its negation) with at most one positive literal (also written as implication), are sufficient to represent and reason about knowledge that the three businesses levels presented in Fig. 1 use during argumentative conversations.

The complexity of playing the games can be reduced to the complexity of deciding about the next move given the previous move and the content of the agent's knowledge base and the shared commitment store. From the game specification, deciding the next move is argumentation-based with a polynomial complexity. Combining the different games is clearly polynomial as the last replied move decides about the game to be played. Therefore, given the fact that businesses/agents' knowledge bases are finite and repeated games with the same content is prohibited, the complexity of the protocol $\mathcal{B} 2 \mathcal{B}-\mathcal{P} \mathcal{P}$ is polynomial.

\section{Case Study}

\subsection{Description and Analysis}

Our running example (provided by IBM Research Division) illustrates an online purchase-order application using Web services technology [24] (Fig. 4). This application, widely used in $\mathcal{B} 2 \mathcal{B}$ settings and supply chain management, is inspired by SpendMap Purchase Order Software ${ }^{3}$. A customer places an order for products via Cust omer-WS (WS for Web service). Based on this order, Customer-WS obtains details on the

\footnotetext{
${ }^{3}$ www.spendmap.com/page.asp?intNodeID=967\&intPageID=1055\&slogon=Bpurch.
} 
customer's purchase history from CRM-WS (Customer Relationship Management) of $\mathcal{B}$ usiness $\mathcal{B}_{1}$. Next, Customer-WS forwards these details to $\mathcal{B}_{1}$ 's Billing-WS, which calculates the customer's bill based on these details (e.g., considering if the customer is eligible for discounts) and sends the bill to CRM-WS. This latter prepares the detailed purchase order based on the bill and sends Inv-Mgmt-WS (Inventory Management) of $\mathcal{B}_{1}$ this order for fulfillment. For those products that are in stock, Inv-Mgmt-WS sends Shipper-WS of $\mathcal{B}_{2}$ a shipment request. Shipper-WS is now in charge of delivering the products to the customer. For those not in stock, Inv-Mgmt-WS sends Supplier-WS of $\mathcal{B}_{3}$ a supply message to the requisite, which provides the products to Shipper-WS for subsequent shipment to the customer.

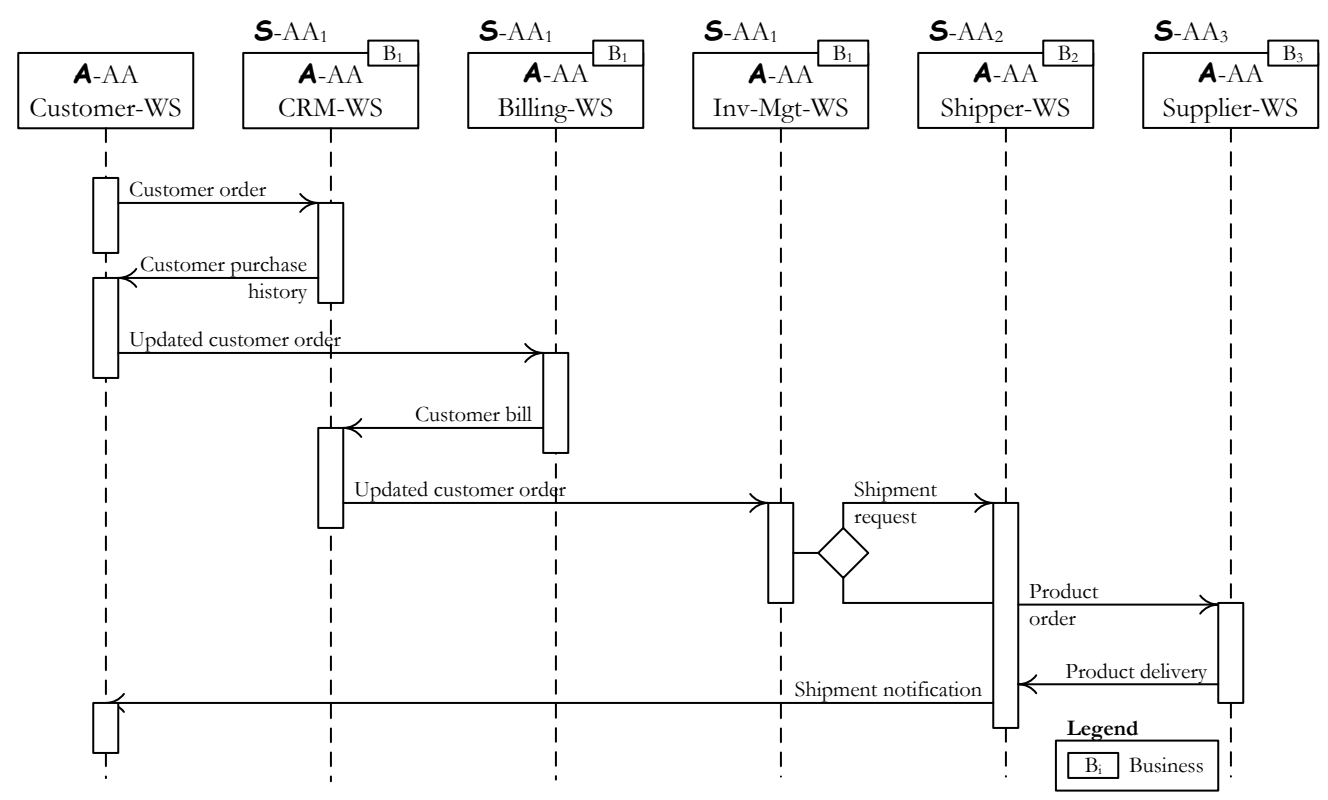

Figure 4. Specification of the purchase-order scenario

From a $\mathcal{B} 2 \mathcal{B}$ perspective, the above application shows how different businesses need to collaborate in order to fulfill customers' needs, as per the three horizontal relations of Fig. 1. This application could be affected by several types of conflicts. For example, $\mathcal{B}_{2}$ 's Shipper-WS may not deliver the products as agreed with $\mathcal{B}_{1}$ 's Inv-Mgmt-WS, perhaps due to lack of trucks. This is an application-level conflict that needs to be resolved using our $\mathcal{B} 2 \mathcal{B}-\mathcal{P} \mathcal{P}$ by which, Shipper-WS tries to persuade Inv-Mgmt-WS about the new shipment time and then, inform Customer-WS of the new delivery time. If not, Shipper-WS may change its policies by cancelling its partnership agreements without prior notice. This is a strategic-level conflict, that calls for either asking $\mathcal{B}_{2}$ to which Shipper-WS belongs to review its policies, or if that does not work, selecting an alternate shipper.

Let $\alpha_{B_{1}}$ be the $\mathcal{A}$-AA of Inv-Mgmt-WS and $\beta_{B_{2}}$ be the $\mathcal{A}$-AA of Shipper-WS. The resolution of the application level conflict along with the use of dialogue games are hereafter provided: 
1. $\beta_{B_{2}}$ identifies the conflict and plays the Initial game by asserting an acceptable argument $a$ about lack of trucks from its $\Sigma_{\beta_{B_{2}}}$ supporting its position: $A \operatorname{ssert}\left(\beta_{B_{2}}, \alpha_{B_{1}}, a\right)$.

2. $\alpha_{B_{1}}$ has an argument $b$ attacking $\beta_{B_{2}}$ 's argument, which is about the available trucks that can be borrowed from a company and could be used to ship the products. $\alpha_{B_{1}}$ plays then the Assertion game by advancing the Attack move: $\operatorname{Attack}\left(\alpha_{B_{1}}, \beta_{B_{2}}, b\right)$.

3. $\beta_{B_{2}}$ replies by playing the Attack game. Because it does not have an argument to change the status of the persuasion topic, but has a partial argument for that, which is about the high price of these particular trucks that could be not accepted by $\alpha_{B_{1}}$, it advances the move: Question $\left(\beta_{B_{2}}, \alpha_{B_{1}}, x\right)$ where $x$ represents accepting or not the new prices. The idea here is that $\beta_{B_{2}}$ can attack $\alpha_{B_{1}}$, if it refuses the new prices that others have accepted.

$\alpha_{B_{1}}$ plays the Question game and answers the question by asserting an argument $c$ in favor of the increased shipment charges: $\operatorname{Assert}\left(\alpha_{B_{1}}, \beta_{B_{2}}, c\right)$.

4. $\beta_{B_{2}}$ plays the Assertion game, and from $\Sigma_{\beta_{B_{2}}} \cup C S_{\alpha_{B_{1}}}$, it accepts the argument and agrees to deliver the products as per the agreed schedule with the new price, which is represented by $d$ : $\operatorname{Accept}\left(\beta_{B_{2}}, \alpha_{B_{1}}, d\right)$. Consequently, the persuasion terminates successfully by resolving the conflict.

Figs. 5 and 6 illustrate the scenario details with the exchanged arguments.

\subsection{Implementation}

We have implemented a proof-of-concept prototype of our $\mathcal{B} 2 \mathcal{B}$ framework using Jadex Agent System (a Java-based programming language for autonomous agents) and applied it to the case-study scenario. Fig. 7 depicts a screenshot of the prototype illustrating the involved agents and computation of the arguments in the scenario. The two involved agents in this scenario (described in Fig. 6) are Inv_Mgmt and Shipper. The console output shows the different computed arguments and their extensions.

Computing the different argument extensions and acceptable arguments and partial arguments is an essential part of our framework and thus, the prototype. This process has been implemented using InterProlog, an open source Java front-end and functional enhancement for Prolog. Because both Jadex Agent System and InterProlog are Javabased, they are compatible and can communicate. The computed arguments and their extensions are then conveyed by InterProlog to Jadex Agent System that implements the $\mathcal{B} 2 \mathcal{B}$ application. The Input Screen in Fig. 8-a is a Java Swing GUI used to specify the agents'knowledge bases, their partial arguments, and the conflicts between arguments. Then, the acceptable arguments and the way to resolve the conflict in the $\mathcal{B} 2 \mathcal{B}$ setting is shown after running the system as illustrated in Fig. 8-b. The arguments used in the figure are taken from the case study where the meaning of every argument is defined in a separate text file, for example argument ${ }^{\prime} a$ is about lack of trucks, which implies delayed delivery.

In this particular running example, agent Inventory (Inv_Mgmt) has two arguments ${ }^{\prime} b^{\prime}$ and ${ }^{\prime} c^{\prime}$ in the knowledge base, which are not attacked. In addition, argument ${ }^{\prime} b^{\prime}$ attacks ${ }^{\prime} a$ ' and argument ${ }^{\prime} e^{\prime}$ attacks ' $b$ ' (the symbol ' $\# n^{\prime}$ is used as a separator). The final text field is for support; for instance support for ' $x^{\prime}$ is ' $c^{\prime}$ (this is captured in the framework by 


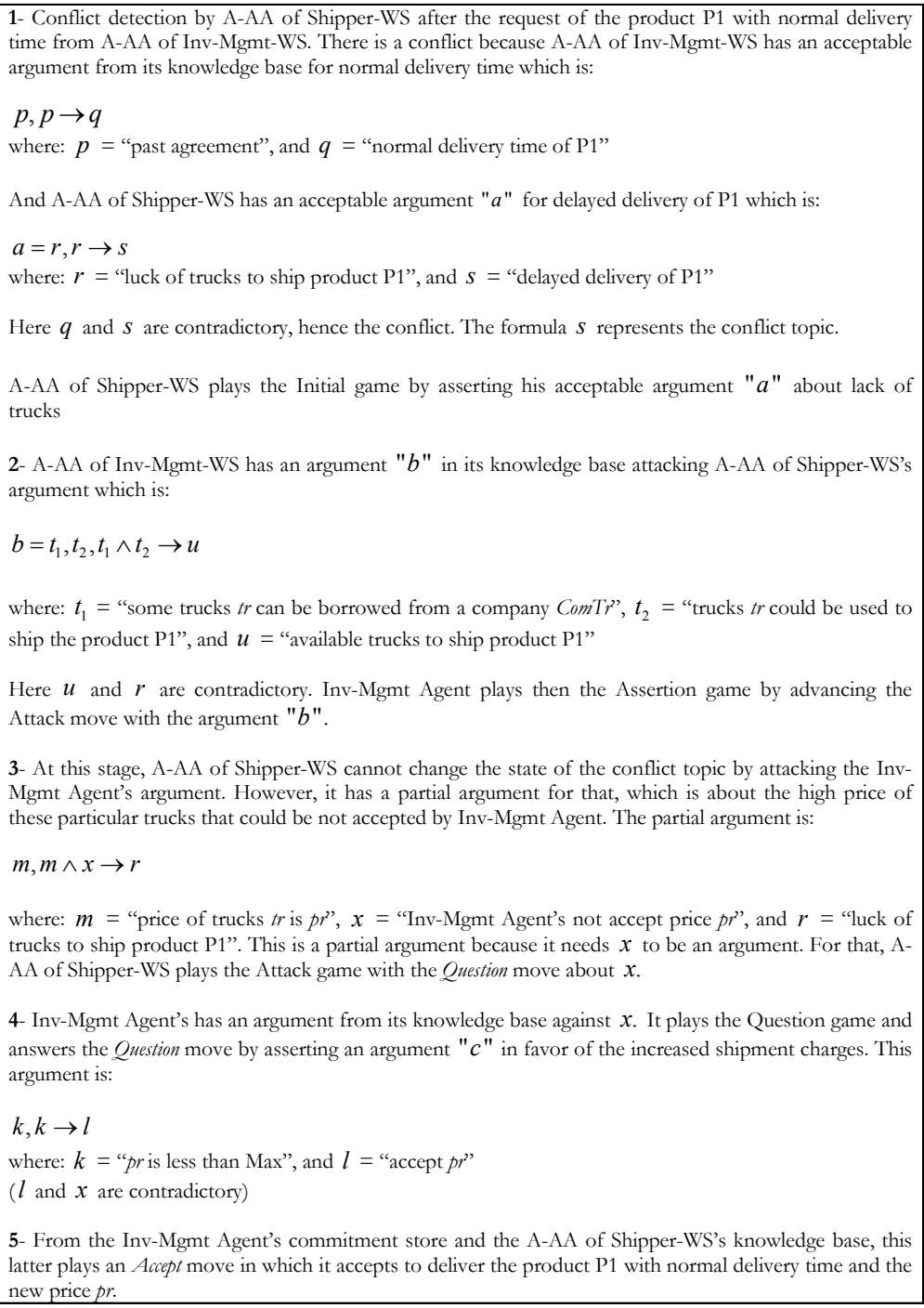

5- From the Inv-Mgmt Agent's commitment store and the A-AA of Shipper-WS's knowledge base, this latter plays an Accept move in which it accepts to deliver the product P1 with normal delivery time and the new price $p r$.

Figure 5. Scenario description

the assertion in Question game). The process of computing the extensions and resolving the conflicts is illustrated in Fig. 9. In this figure, (1) check_partial, (2) check_ques, (3) Find_Extension, and (4) ques_ans are Java methods having the following respective purposes: (1) filter partial arguments to return only arguments; (2) ask for missing information; (3) find grounded or preferred extensions from the argumentation framework; and (4) find support for information asked. 


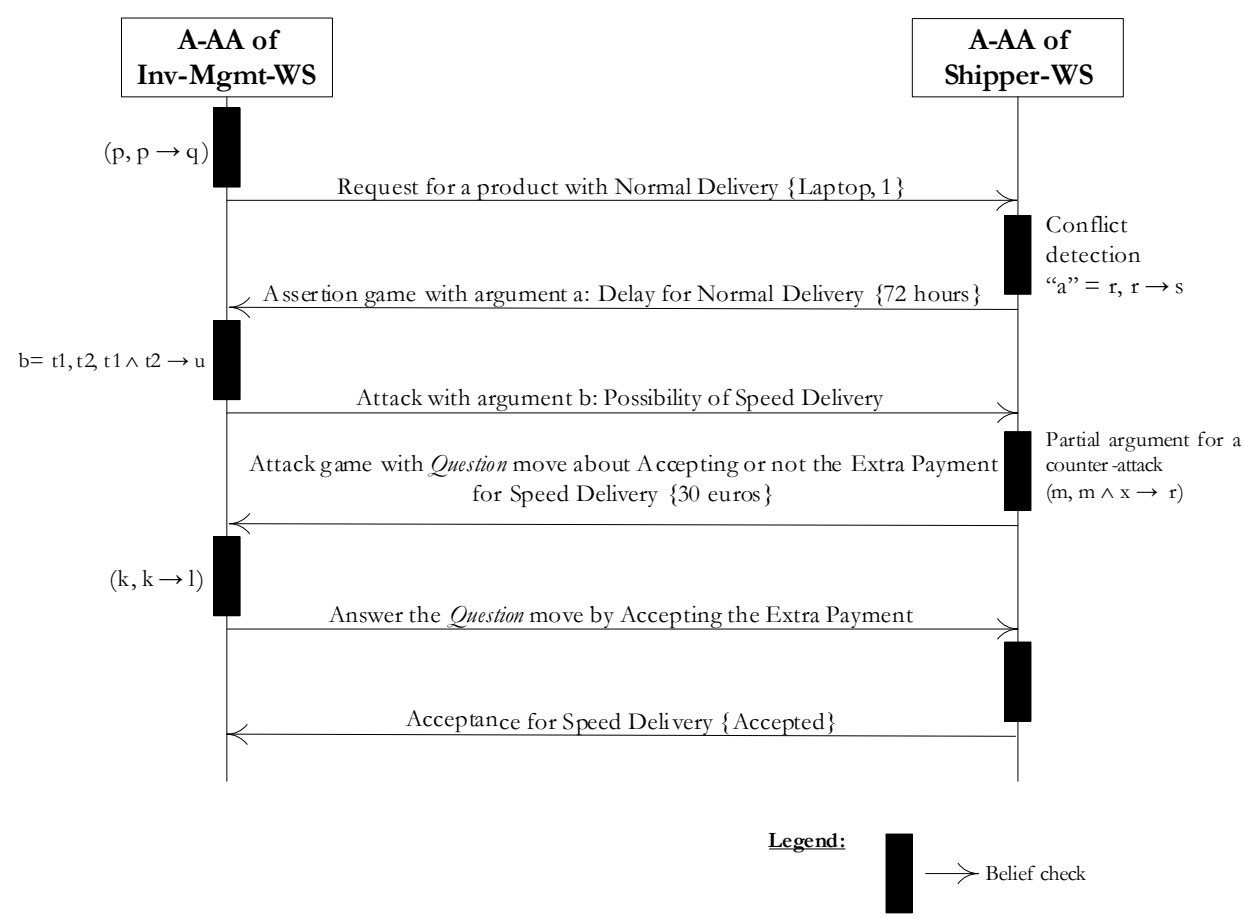

Figure 6. Sample of interactions between $\mathcal{A}$-AA of Inv-Mgmt-WS and $\mathcal{A}$-AA of Shipper-WS

\section{Related Work}

Recent years have seen a continuing surge of interest in designing and deploying $\mathcal{B} 2 \mathcal{B}$ applications. Service-oriented architecture is the most widely methodology that have been used in this field $[15,19,26,28]$. In [15] the author proposes the exploitation of Web services and intelligent agent techniques for the design and development of a $\mathcal{B} 2 \mathcal{B}$ eCommerce application. A multi-party multi-issue negotiation mechanism is developed for this application. This negotiation is a Pareto optimal negotiation based on game theory. This proposal aims at achieving an agrement by computing concessions and generating offers in order to maximize the utility of the participating agents. However, unlike our argumentation-based framework, this mechanism cannot be used to resolve general conflicts as those discussed in Sections 2 and 3. In [19], the authors develop a methodology for $\mathcal{B} 2 \mathcal{B}$ design applications using Web-based data integration. The aim is the creation of adaptable semantics oriented meta-models to facilitate the design of mediators by considering several characteristics of interoperable information systems such as extensibility and composability. The methodology is used to build cooperative environments involving the integration of Web data and services. Unlike our methodology, this proposal does not consider conflicts that can arise during the cooperation phase and only addresses the cooperation from technological point of view.

On the other side, and from an argumentation viewpoint, some interesting protocols for persuasion and inquiry have been proposed. [2] propose Persuasive Argument for Multiple Agents (PARMA) Protocol, which enables participants to propose, attack, 


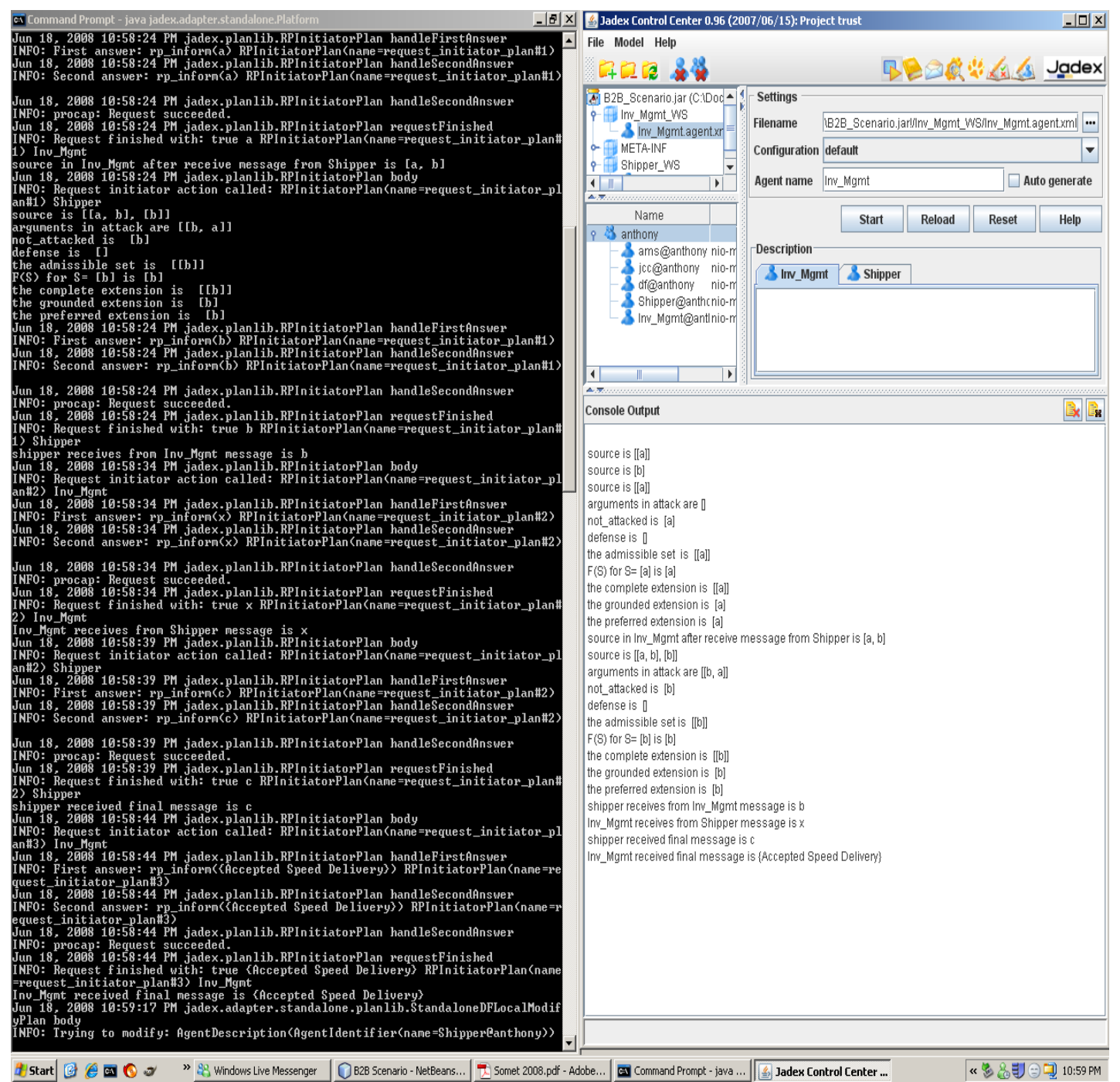

Figure 7. A screenshot from the prototype -involved agents and computing arguments-

and defend an action or course of actions. This protocol is specified using logical consequence and denotational semantics. The focus of this work is more on the semantics of the protocol rather than the dynamics of interactions. [7] propose a dialogue-game inquiry protocol that allows two agents to share knowledge in order to construct an argument for a specific claim. There are many fundamental differences between this protocol and ours. Inquiry and persuasion settings are completely different since the objectives and dynamics of the two dialogues are different. In [7], argumentation is captured only by the notion of argument with no attack relation between arguments. This is because agents collaborate to establish joint proofs. However, in our system, agents can reason about conflicting assumptions, and they should compute different acceptability seman- 


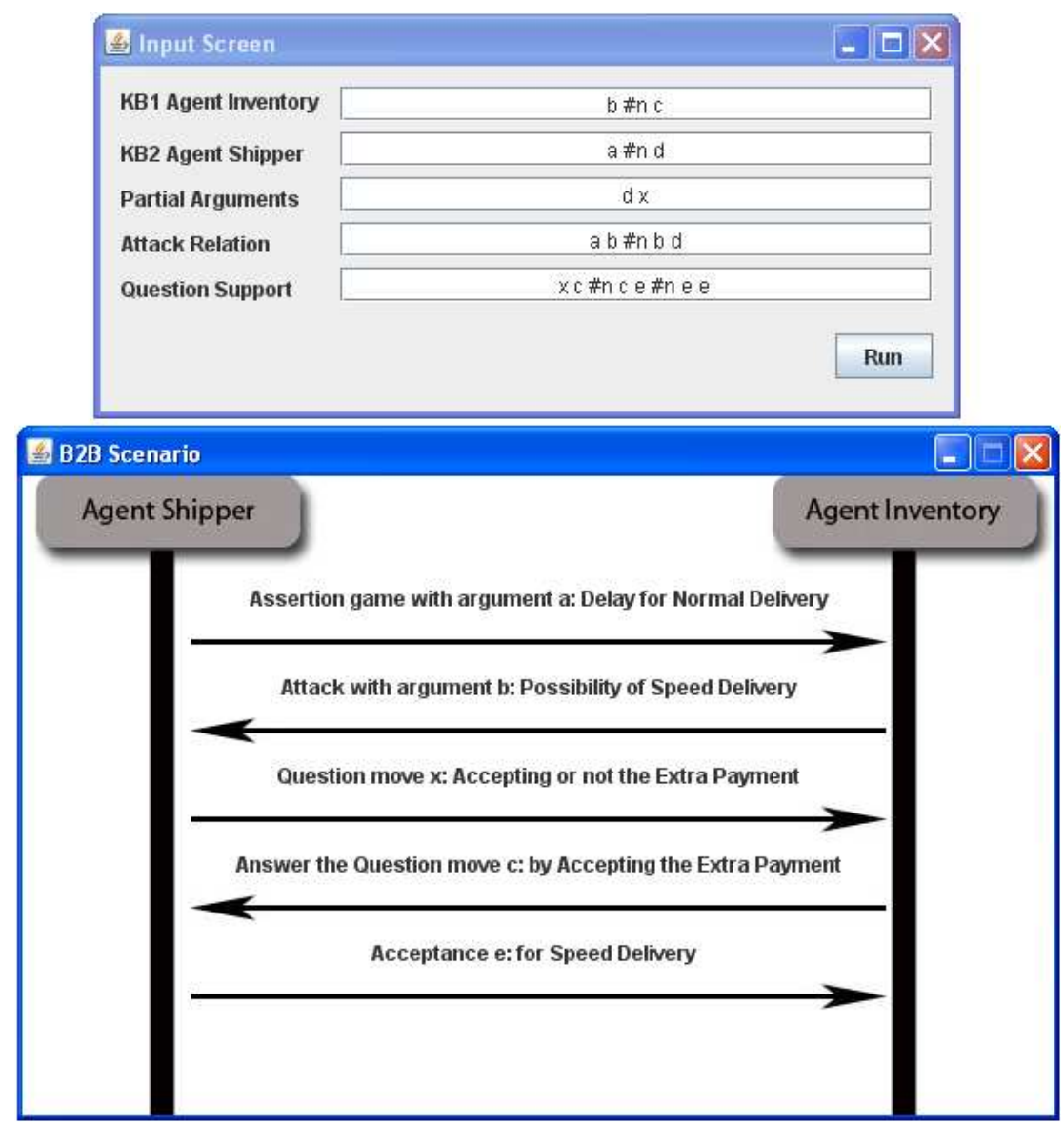

Figure 8. a- Input screen, b- Resolving conflict scenario

tics, not only to win the dispute, but also to reason internally in order to remove inconsistencies from their assumptions. From the specification perspective, there are no similarities between the two protocols. Our protocol is specified as a set of rules about which agents can reason using argumentation, which captures the agents' choices and strategies. However, in [7] the protocol is specified in a declarative manner and the strategy is only defined as a function without specifying how the agents can use it. The adopted moves in the two proposals are also different. Another technical, but fundamental difference in the two protocols is the possibility in our protocol of considering not only the last uttered argument, but any previous argument which allows agents to consider and try different ways of attacking each other.

\section{Conclusions and Future Work}

In this paper, a 3-level framework for $\mathcal{B} 2 \mathcal{B}$ applications is presented. The three levels namely strategic, application and resource are populated with argumentative agents. The 


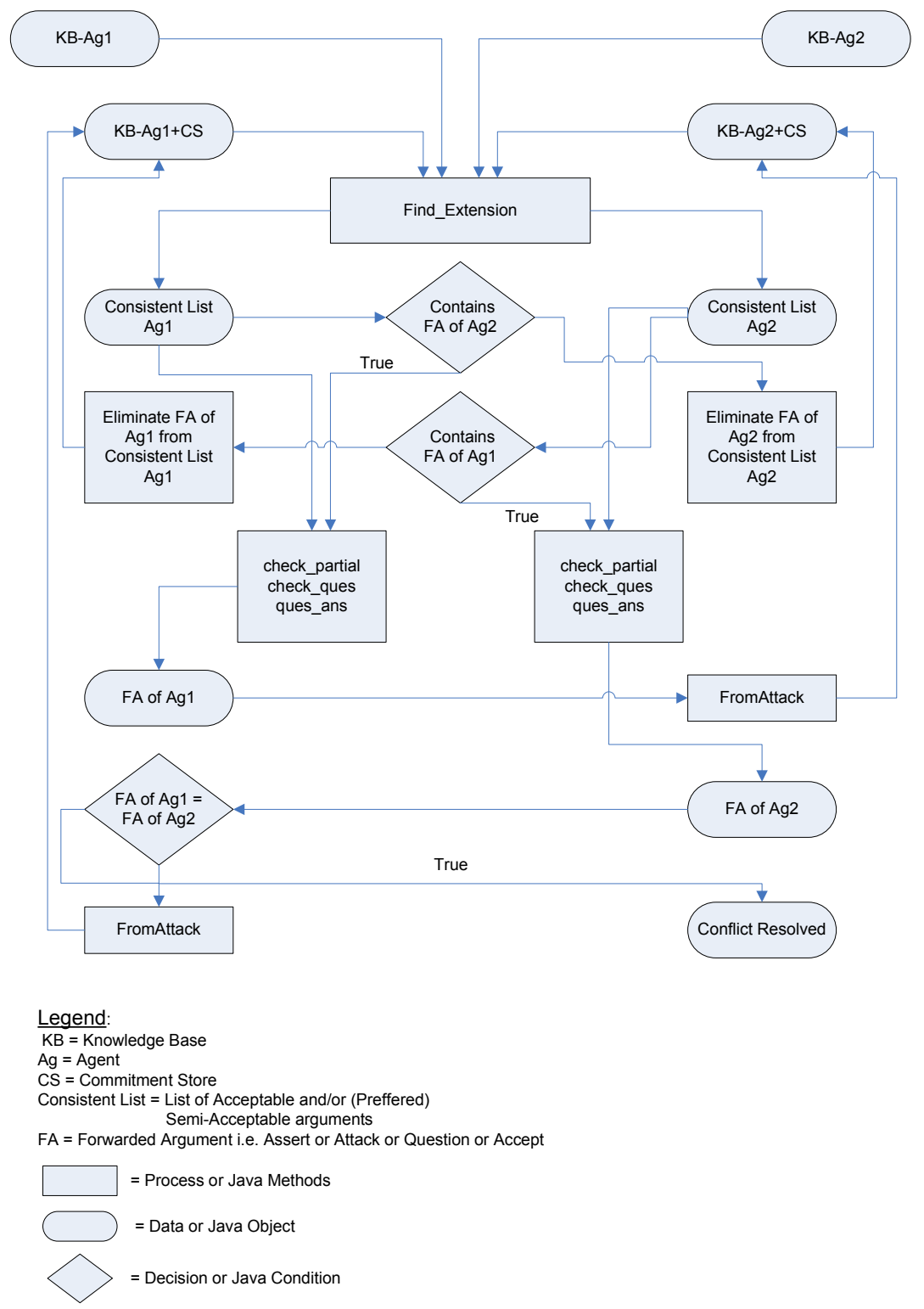

Figure 9. Process of computing the extensions and resolving the conflicts

first contribution of this paper is the development of a framework to set-up collaborations among autonomous businesses (via strategic level), and execute and manage these collaborations (via application level). Inevitably, given the autonomous and dynamic nature of businesses, conflicts are bound to arise. The second contribution is the proposition of a sound and complete persuasion protocol for resolving such conflicts. This protocol, that includes inquiry stages is argumentation-based.

Future work would include considering a negotiation protocol during argumentation 
and scaling up and demonstrating our argumentation-based model on larger examples. Additional research venues would be enriching this model with fuzzy concepts and new criteria of acceptability and with contextual ontologies when modeling knowledge bases of individual agents. Promoting truth telling in our argumentation-based framework and considering non-cooperative agents are other plans for future investigation.

\section{Acknowledgements}

The first author is partially supported by Natural Sciences and Engineering Research Council of Canada (NSERC), Fonds québécois de la recherche sur la nature et les technologies (NATEQ), and Fonds québécois de la recherche sur la société et la culture (FQRSC). The authors would also like to thank the reviewers for their very helpful comments and suggestions.

\section{References}

[1] L. Amgoud, Y. Dimopoulos, and P. Moraitis. A Unified and Genaral Framwork for Argumentationbased Negotiation. In The International Conference on Autonomous Agents and Multiagent Systems. ACM Press, 2007.

[2] K. Atkinson, T. Bench-Capon, and P. McBurney. A Dialogue Game Protocol for Multi-Agent Argument over Proposals for Action. Journal of Autonomous Agents and Multi-Agent Systems, 11(2), 2005.

[3] T. Bench-Capon and P. Dunne. Argumentation in Artificial Intelligence. Artificial Intelligence, 171(1015), 2007.

[4] J. Bentahar, Z. Maamar, D. Benslimane, and P. Thiran. An Argumentation Framework for Communities of Web Services. IEEE Intelligent Systems, 22(6), 2007.

[5] J. Bentahar, B. Moulin, and M. Bélanger. A Taxonomy of Argumentation Models used for Knowledge Representation. Artificial Intelligence Review, DOI: 10.1007/s10462-010-9154-1, 2010.

[6] J. Bentahar, F. Toni, J.-J. Meyer, and J. Labban. A Security Framework for Agent-based Systems. Journal of Web Information Systems, 3(4):341-362, 2007.

[7] E. Black and A. Hunter. A Generative Inquiry Dialogue System. In The International Conference on Autonomous Agents and Multiagent Systems. ACM Press, 2007.

[8] A. Bondarenko, P. Dung, R. Kowalski, and F. Toni. An Abstract, Argumentation-Theoretic Approach to Default Reasoning. Artificial Intelligence, 93(1-2):63-101, 1997.

[9] P. Dung. The Acceptability of Arguments and its Fundamental Role in Non-Monotonic Reasoning and Logic Programming and n-Person Game. Artificial Intelligence, 77:321-357, 1995.

[10] P. Dung, P. Mancarella, and F. Toni. Computing ideal sceptical argumentation. Artificial Intelligence, Special Issue on Argumentation in Artificial Intelligence, 171(10-15):642-674, 2007.

[11] H. Fujita. Special Issue on "Techniques to Produce Intelligent_Secure Software". Knowl.-Based Syst., 20(7):614-616, 2007.

[12] A. Garcia and G. Simari. Defeasible Logic Programming: an Argumentative Approach. Theory and Practice of Logic Programming, 4(1):95-138, 2004.

[13] V. Gruhn and H. Fujita. Special Issue on "Intelligent Software Design”. Knowl.-Based Syst., 19(2):105106,2006

[14] B. Ktari, H. Fujita, M. Mejri, and D. Godbout. Toward a New Software Development Environment. Knowl.-Based Syst., 20(7):683-693, 2007.

[15] R. Lau. Towards a Web Services and Intelligent Agents-based Negotiation System for B2B eCommerce. Electronic Commerce Research and Appl., 6(3), 2007.

[16] N. Maudet and B. Chaib-draa. Commitment-based and Dialogue Game-based Protocols, new trends in agent communication languages. Knowledge Engineering Review, 17(2):157-179, 2002.

[17] P. McBurney and S. Parsons. Games that Agents Play: A Formal Framework for Dialogues between Autonomous Agents. Journal of Logic, Language, and Information, 11(3):315-334, 2002. 
[18] N. Milanovic and M. Malek. Current Solutions for Web Service Composition. IEEE Internet Computing, 8(6), November/December 2004.

[19] C. Nicolle, K. Yétongnon, and J. Simon. XML Integration and Toolkit for B2B Applications. Journal of Database Management, 14(4), 2003.

[20] S. Parsons, M. Wooldridge, and L. Amgoud. On the Outcomes of Formal Inter-Agent Dialogues. In Proceedings of The International Conference on Autonomous Agents and Multiagent Systems. ACM Press, 2003.

[21] P. Pasquier, I. Rahwan, F. Dignum, and L. Sonenberg. Argumentation and Persuasion in the Cognitive Coherence Theory. In The 1st International Conference on Computational Models of Argument. IOS Press, 2006.

[22] H. Prakken. Formal Systems for Persuasion Dialogue. The Knowledge Engineering Review, 24(2), 2005.

[23] I. Rahwan and P. McBurney. Argumentation Technology. IEEE Intelligent Systems, 22(6), 2007.

[24] E. H. Sabri, A. P. Gupta, and M. A. Beitler. Purchase Order Management Best Practices: Process, Technology, and Change Management. J. Ross Publishing, 2006.

[25] F. Sadri, F. Toni, and P. Torroni. Dialogues for Negotiation: Agent Varieties and Dialogue Sequences. In The International Workshop on Agents, Theories, Architectures and Languages, 2001.

[26] Y. Udupi and M. Singh. Contract Enactment in Virtual Organizations: A Commitment-Based Approach. In The 21st National Conference on Artificial Intelligence, 2006.

[27] D. N. Walton and E. C. W. Krabbe. Commitment in Dialogue: Basic Concepts of Interpersonal Reasoning. Suny Series in Logic and Language, 1995.

[28] A. Williams, A. Padmanabhan, and B. Blake. Local Consensus Ontologies for B2B-Oriented Service Composition. In The International Joint Conference on Autonomous Agents and Multiagent Systems, 2003.

\section{Appendix}

Proof of Proposition 3 Without loss of generality, let $a, a_{1}, \ldots, a_{n}$ be arguments in a given argumentation framework such that $a_{1}, \ldots, a_{n}$ are the only attackers of $a$ and $a$ is the only attacker of these arguments. According to Definition 6, the argument $a$ is not acceptable since it is attacked and not defended, directly or indirectly by a non-attacked argument. Because it is defended, $a$ belongs to some preferred extensions. However, $a$ does not belong to all of them. For example, $a$ does not belong to the preferred extension to which the arguments $a_{1}, \ldots, a_{n}$ belong since these arguments belong also to some preferred extensions because they are defended. $a$ is then semi-acceptable.

Proof of Proposition 4 We prove this proposition by a counter example using Example 4 . In this example $\{a, d\}$ and $\{b, d\}$ are complete extensions (preferred extensions). However, $\{d\}$ is not a complete extension.

Proof of Proposition 5 By Definition 5, the grounded extension is included in all preferred extensions. Consequently, using Definition 8, an eliminated argument is not acceptable. Also, according to Definition 7, an eliminated argument is not semi-acceptable and not preferred semi-acceptable.

Proof of Proposition 6 Suppose that $\exists x \in \mathcal{W F}: a_{x}^{p}$ is not acceptable. Therefore, a part of the non-missing part of $a_{x}^{p}$ is not acceptable. Because this part is also a part of $a$, then $a$ is not acceptable. Contradiction!

Proof of Proposition 9 To prove this we should prove that $C_{a s 3} \Rightarrow \neg C_{a s 1} \wedge \neg C_{a s 2}$. Using the logical calculation, we can easily prove that $\neg C_{a s 1} \wedge \neg C_{a s 2}=\neg C_{a s 1} \wedge \neg O p_{a s_{2}}^{q u_{1}} \wedge$ 
$\neg O p_{a s_{2}}^{q u_{2}}$. Also, if an agent $\beta$ can build an acceptable argument $a$ from $\mathcal{A}_{\beta} \cup C S_{\alpha}$, then it cannot build an acceptable or (preferred) semi-acceptable argument attacking $a$ from the same set. Therefore, $\mathcal{A R}\left(\mathcal{A}_{\beta} \cup C S_{\alpha}\right) \triangleright a \Rightarrow \neg C_{a s 1}$. Thus the result follows.

Proof of Theorem 1 Agents' knowledge bases are finite and repeating moves with the same content is prohibited. Consequently, the number of Attack and Question moves that agents can play is finite. At a given moment, agents will have two possibilities only: Accept if an acceptable argument can be built from $C S_{\alpha} \cup C S_{\beta}$, or Stop, otherwise. Therefore, the protocol terminates successfully by Accept, or unsuccessfully by Stop when Accept move cannot be played, which means that only semi-acceptable arguments are included in $C S_{\alpha} \cup C S_{\beta}$.

Proof of Theorem 2 For simplicity and without loss of generality, we suppose that agent $\alpha$ starts the persuasion.

Let us first prove the $\Rightarrow$ direction: $\mathcal{A R}\left(\mathcal{A}_{\alpha} \cup \mathcal{A}_{\beta}\right) \triangleright a \Rightarrow \mathcal{A R}(\cup C S) \triangleright a$.

In the protocol, the persuasion starts when a conflict over $p$ occurs. Consequently, the case where $\mathcal{A}_{\alpha} \triangleright a$ and $\mathcal{A}_{\beta} \triangleright a$ does not hold. The possible cases are limited to three:

1. $\mathcal{A}_{\alpha} \triangleright a$ and $\mathcal{A}_{\beta} \not a$. In this case, agent $\alpha$ starts the persuasion over $p$ by asserting $a$. Agent $\beta$ can either play the Attack move or the Question move. Because $\mathcal{A R}\left(\mathcal{A}_{\alpha} \cup \mathcal{A}_{\beta} \triangleright a\right)$ all the $\beta$ 's arguments will be counter-attacked. For the same reason, $\beta$ cannot play the Stop move. Consequently, at the end, $\beta$ will play an Accept move. It follows that $\mathcal{A R}(\cup C S \triangleright a)$.

2. $\mathcal{A}_{\alpha} \not a$ and $\mathcal{A}_{\beta} \triangleright a$. In this case, agent $\alpha$ starts the persuasion by asserting an acceptable argument $b$ in its knowledge base against $p\left(\mathcal{A}_{\alpha} \triangleright b\right)$. This argument will be attacked by agent $\beta$, and the rest is identical to case 1 by substituting agent roles.

3. $\mathcal{A}_{\alpha} \not a$ and $\mathcal{A}_{\beta} \not a$. To construct argument $a$ out of $\mathcal{A}_{\alpha} \cup \mathcal{A}_{\beta}$, two cases are possible. Either, (1) agent $\alpha$ has an acceptable partial argument $a_{\partial}^{Y}$ for $p$ and agent $\beta$ has the missing assumptions (or some parts of the missing assumptions, and agent $\alpha$ has the other parts), or (2) the opposite (i.e., agent $\beta$ has an acceptable partial argument $a_{\partial}^{Y}$ for $p$ and agent $\alpha$ has the missing assumptions (or some parts of the missing assumptions, and agent $\beta$ has the other parts)). Only the second case is possible since the first one is excluded by hypothesis. For simplicity, we suppose that agent $\alpha$ has all the missing assumptions, otherwise the missing assumptions will be built by exchanging the different partial arguments. Agent $\alpha$ starts the persuasion by asserting an acceptable argument $b$ in its knowledge base against $p$. Agent $\beta$ can either play an Attack or a Question move. If attack is possible, then agent $\alpha$ can either counter-attack or play the Stop move. The same scenario continues until agent $\alpha$ plays Stop, and then agent $\beta$ plays a Question Move. Agent $\alpha$ answers now the question by providing the missing assumptions, after which agent $\beta$ attacks and agent $\alpha$ can only accept since $\mathcal{A R}\left(\mathcal{A}_{\alpha} \cup \mathcal{A}_{\beta} \triangleright a\right)$. It follows that $\mathcal{A R}(\cup C S \triangleright a)$.

Let us now prove the $\Leftarrow$ direction: $\mathcal{A R}(\cup C S) \triangleright a \Rightarrow \mathcal{A R}\left(\mathcal{A}_{\alpha} \cup \mathcal{A}_{\beta}\right) \triangleright a$.

In the protocol, to have $\mathcal{A R}(\cup C S) \triangleright a$ one of the two agents, say agent $\alpha$, puts forward the argument $a$ and the other, agent $\beta$, accepts it. On the one hand, to advance an argument, agent $\alpha$ plays the Assert move (in the initial or question rules) or Attack 
move (in the assertion or attack rules). In all these cases, we have: $\mathcal{A R}\left(\mathcal{A}_{\alpha} \cup C S_{\beta}\right) \triangleright a$ and there is no partial acceptable argument attacking $a$ from $\mathcal{A}_{\alpha} \cup S C_{\beta}$. On the other hand, to accept an argument (in the assertion or attack rules), agent $\beta$ should check that $\mathcal{A R}\left(\mathcal{A}_{\beta} \cup C S_{\alpha}\right) \triangleright a$, there is no other arguments changing the status of the persuasion topic, and there is no partial acceptable argument attacking $a$ from $\mathcal{A}_{\beta} \cup S C_{\alpha}$. Therefore we obtain: $\mathcal{A R}\left(\mathcal{A}_{\alpha} \cup C S_{\beta} \cup \mathcal{A}_{\beta} \cup C S_{\alpha}\right) \triangleright a$. Because $C S_{\alpha} \subseteq \mathcal{A}_{\alpha}$ and $C S_{\beta} \subseteq \mathcal{A}_{\beta}$ we are done.

Proof of Theorem 3 Suppose for simplicity reasons that the agents' knowledge bases contain directly arguments and partial arguments. The following is a counterexample proving that if the agents are not truth telling, the protocol will not be sound and complete. Suppose that agent $\alpha$ starts the protocol such that: $\Sigma_{\alpha}=\{b, c, x\}, \Sigma_{\beta}=\left\{a, d_{x}^{p}\right\}$, $\mathcal{A T}(a, b), \mathcal{A} \mathcal{T}(c, a), \mathcal{A} \mathcal{T}(d, c), C S_{\alpha}=\{b, c, \bar{x}\}, C S_{\beta}=\left\{a, d_{x}^{p}\right\}$. In this example, after playing the Question move about $x$ by agent $\beta$, agent $\alpha$ answers by asserting $\bar{x}$, instead of $x$. We have then $\mathcal{A R}(\cup C S) \triangleright b \wedge \mathcal{A R}\left(\Sigma_{\alpha} \cup \Sigma_{\beta}\right) \not b$ (the protocol is not sound) and $\mathcal{A R}\left(\Sigma_{\alpha} \cup \Sigma_{\beta}\right) \triangleright a \wedge \mathcal{A R}(\cup C S) \not a$ (the protocol is not complete). 